\title{
Inflation and leptogenesis in high-scale supersymmetry
}

\author{
Kunio Kaneta $\odot,{ }^{1}$ Yann Mambrini, ${ }^{2}$ Keith A. Olive, ${ }^{1}$ and Sarunas Verner ${ }^{1}$ \\ ${ }^{1}$ William I. Fine Theoretical Physics Institute, School of Physics and Astronomy, University of Minnesota, \\ Minneapolis, Minnesota 55455, USA \\ ${ }^{2}$ Laboratoire de Physique Théorique Université Paris-Sud, F-91405 Orsay, France
}

(Received 16 November 2019; published 7 January 2020)

\begin{abstract}
No-scale supergravity provides a successful framework for Starobinsky-like inflation models. Two classes of models can be distinguished depending on the identification of the inflaton with the volume modulus, $T$ (C models), or a matterlike field, $\phi$ (WZ models). When supersymmetry is broken, the inflationary potential may be perturbed, placing restrictions on the form and scale of the supersymmetry breaking sector. We consider both types of inflationary models in the context of high-scale supersymmetry. We further distinguish between models in which the gravitino mass is below and above the inflationary scale. We examine the mass spectra of the inflationary sector. We also consider, in detail, mechanisms for leptogenesis for each model when a right-handed neutrino sector, used in the seesaw mechanism to generate neutrino masses, is employed. In the case of $\mathrm{C}$ models, reheating occurs via an inflaton decay to two Higgs bosons. However, there is a direct decay channel to the lightest right-handed neutrino which leads to nonthermal leptogenesis. In the case of WZ models, in order to achieve reheating, we associate the matterlike inflaton with one of the right-handed sneutrinos whose decay to the lightest right-handed neutrino simultaneously reheats the Universe and generates the baryon asymmetry through leptogenesis.
\end{abstract}

DOI: 10.1103/PhysRevD.101.015002

\section{INTRODUCTION}

There are many motivations for supersymmetry including the solution to the hierarchy problem [1], gauge coupling unification [2], the stability of the Higgs vacuum [3], radiative electroweak symmetry breaking [4], and viable dark matter candidates [5]. Supersymmetry also aids in the construction of inflationary models [6], allowing naturally for flat directions suitable for inflation and keeping radiative corrections in check. Indeed, the natural framework for formulating supersymmetric models of inflation is that of supergravity [7]. However, generic supergravity models often induce what is known as the $\eta$ problem [8], which is easily addressed in a no-scale supergravity framework $[9,10]$.

It is remarkable that the Starobinsky model based on $R+R^{2}$ gravity [11,12], which was one of the first models of inflation, is in excellent agreement with the most recent Planck measurements [13] of the tensor-to-scalar ratio $r=$ 0.0035 and the tilt of the scalar perturbations $n_{s}=0.965$. The Starobinsky scalar potential for a canonically normalized inflaton field, $x$, is given by

Published by the American Physical Society under the terms of the Creative Commons Attribution 4.0 International license. Further distribution of this work must maintain attribution to the author(s) and the published article's title, journal citation, and DOI. Funded by SCOAP.

$$
V=\frac{3}{4} m^{2}\left(1-e^{-\sqrt{\frac{2}{3}} x}\right)^{2}
$$

and can be easily realized in no-scale supergravity [14-30]. These models must contain at least two chiral fields, which we will denote as $T$, a volume modulus, and $\phi$, a matterlike field [15]. The nonminimal Kähler potential with two chiral fields is expressed as

$$
K=-3 \ln \left(T+\bar{T}-\frac{|\phi|^{2}}{3}\right)
$$

parametrizing a noncompact $\frac{S U(2,1)}{S U(2) \times U(1)}$ coset manifold [14] The inflationary models can be divided into two classes, in which either the volume modulus $T$ or the matterlike field $\phi$ is identified as the inflaton $[15,18]$. Depending on the specific model, an additional chiral multiplet may be necessary to break supersymmetry [26,27].

The scale of supersymmetry breaking is usually assumed to be near the weak scale. In that case, one easily resolves the issues that motivate supersymmetry in the first place. However, with the exception of the hierarchy problem, the problems discussed in the beginning of this section can also be resolved in the context of high-scale supersymmetry. For example, gauge coupling unification in high-scale supersymmetry has been shown to be effective in $\mathrm{SO}(10)$ models of grand unification [31]. To be more precise, it is known that gauge coupling unification also occurs in 
nonsupersymmetric models $\mathrm{SO}(10)$ models when the unified gauge symmetry is broken down to the Standard Model (SM) gauge group through an intermediate scale gauge group [32-36]. Similarly, the stability of the Higgs vacuum can be maintained in both high-scale supersymmetry [31] and nonsupersymmetric models [36], when an additional scalar field below $10^{10} \mathrm{GeV}$ is present (which can also drive radiative electroweak symmetry breaking).

Among the best studied candidates for dark matter are those arising in weak scale supersymmetric models [5]. In this context, R-parity conservation renders the lightest supersymmetric particle (LSP) stable. Most phenomenological studies favor neutralino dark matter models, but models with a gravitino LSP have also been considered [5,37-45]. However, both the detection of supersymmetric particles at the LHC [46] and dark matter direct-detection experiments searching for neutralino dark matter, such as LUX [47], PandaX-II [48], and XENON1T [49], remain elusive.

It is possible that the supersymmetry breaking scale is beyond the reach of the LHC, and that the corresponding scattering cross sections for multi- $\mathrm{TeV}$ neutralinos are below the current detection limits, as is the case in some variants of the constrained minimal supersymmetric standard model (CMSSM) [50]. Alternatively, supersymmetry breaking may occur at the $\mathrm{PeV}$ scale as in models of pure gravity mediation with a wino or Higgsino dark matter candidate [51,52].

These considerations motivate us to explore models with high-scale supersymmetry breaking [31,53]. Indeed, it is possible to construct viable models with a significantly higher supersymmetry breaking scale so that all the superpartners, except for the gravitino, lie above the inflationary scale [27,54-56]. In this case, the gravitino with a mass of order $m_{3 / 2} \gtrsim 0.1 \mathrm{EeV}$ may play the role of dark matter. Its production occurs through the reheating process after inflation [5,38,57-62]. In weak-scale supersymmetry, single gravitinos together with other supersymmetric particles can be produced from scattering processes, e.g., gluon + gluon $\rightarrow$ gravitino + gluino. However, in highscale supersymmetry models, gravitinos must be produced in pairs [63], and this process is highly sensitive to the maximum temperature. Therefore, to obtain the correct gravitino relic density, we require a relatively high reheating temperature $T_{\mathrm{RH}} \gtrsim 10^{10} \mathrm{GeV}$.

It is also important to note that the existence of dark matter in nonsupersymmetric $\mathrm{SO}(10)$ models is also possible when the intermediate scale gauge group is broken via a 126-dimensional representation as a $Z_{2}$ discrete symmetry (similar to $R$ parity) is preserved $[33-36,65,66]$.

\footnotetext{
${ }^{1}$ In fact, once noninstantaneous reheating is considered [64], it is the maximum temperature attained that gives the largest contribution to the dark matter abundance.
}

In this paper, we consider high-scale supersymmetry models in conjunction with no-scale Starobinsky-like models of inflation [27]. We discuss inflationary models based on a noncompact $\frac{S U(2,1)}{S U(2) \times U(1)}$ Kähler potential (2), where either the volume modulus $T$ or a matterlike field $\phi$ is driving inflation. The two types of models are distinguished by their couplings to the Standard Model (which leads to different reheating mechanisms [26]) and the supersymmetry breaking sector. Supersymmetry breaking can perturb the inflationary potential, making it no longer viable for inflation. This is particularly the case when supersymmetry breaking is pushed to very high scales. Supersymmetry breaking also affects particle mass spectrum in the inflationary/SUSY breaking sector. We explore both of these effects in the models discussed below.

We extend our high-scale supersymmetry framework and incorporate various models of leptogenesis $[67,68]$. This is accomplished by introducing a right-handed neutrino sector. The small left-handed neutrino masses are obtained via the classical seesaw mechanism [69], which leads to lepton number violation. Most importantly, the decay of the heavy right-handed neutrinos into Higgs bosons and leptons produce a lepton asymmetry, which is subsequently converted to a baryon asymmetry by sphaleron transitions $[70,71]$. In this paper, we focus on models of nonthermal leptogenesis [67]. In this case, the inflaton decays directly to a right-handed neutrino which is out-of-equilibrium if its mass is larger than the reheating temperature $T_{\mathrm{RH}}$. The subsequent out-of-equilibrium decay of the right-handed neutrino then produces the lepton asymmetry.

The structure of this paper is as follows. We first review how the Starobinsky-like inflation models arise in no-scale supergravity. In Sec. III, we review the basics of leptogenesis as needed in our inflationary context. We then consider separately the case where the inflaton is associated with the $T$ field (Sec. IV) or with $\phi$ (Sec. VI). Within each case, we distinguish models in which the gravitino mass is below and above the inflationary scale. We take into account the effects of supersymmetry breaking on the inflationary potential and the particle mass spectrum and show that the production of inflatinos is not problematic. Furthermore, in each case, we discuss the mechanism for reheating, leptogenesis, and dark matter (in Secs. V and VII). Our conclusions are given in Sec. VIII.

\section{NO-SCALE STAROBINSKY MODELS OF INFLATION}

The Starobinsky model of inflation can be realized in a no-scale supergravity framework by considering the Kähler potential form, given by Eq. (2), and combining it with a specific choice of a superpotential. If we consider the Cecotti superpotential form [72],

$$
W_{\mathrm{C}}=\sqrt{3} m \phi\left(T-\frac{1}{2}\right),
$$


where the inflaton is associated with the volume modulus $T$. When the vacuum expectation value of a matterlike field is fixed to $\langle\phi\rangle=0$ by introducing the higher-order stabilization terms in the Kähler potential (2) [15,73], as discussed later in this section, we obtain the Starobinsky inflationary potential (1) in terms of the canonically normalized field $x$, given by the field redefinition, $T=\frac{1}{2} e^{\sqrt{2 / 3} x}$.

Similarly, if we consider the Wess-Zumino form for the superpotential [14],

$$
W_{\mathrm{WZ}}=m\left(\frac{\phi^{2}}{2}-\frac{\phi^{3}}{3 \sqrt{3}}\right)
$$

where the inflaton is associated with a matterlike field $\phi$, and we stabilize the volume modulus dynamically at its vacuum expectation value of $\langle T\rangle=\frac{1}{2}$, the Starobinsky inflationary potential is obtained by making the canonical field redefinition, $\phi=\sqrt{3} \tanh (x / \sqrt{6})$.

In both models, the scale of inflation is characterized by a single mass scale $m$, which determines the amplitude of density fluctuations, $A_{s} \simeq 2 \times 10^{-9}$, as measured by Planck [13]. For $N_{*}=55$, where $N_{*}$ is the number of $e$-foldings before the end of inflation, the mass scale corresponds to $m \simeq 1.2 \times 10^{-5} M_{P} \simeq 3 \times 10^{13} \mathrm{GeV}$ [14], and we use this value throughout this paper. $^{2}$ If we combine the Kähler potential (2) with either of the superpotential forms (3) or (4), we find that the parameter $m$ can be identified with the mass of the canonically normalized inflaton field. Therefore, in some models of leptogenesis, it seems natural to identify the inflaton with one of the right-handed sneutrinos [16], which then decays into right-handed neutrinos responsible for leptogenesis.

In fact, the two models listed above are simply two examples of a wide class of superpotential models, which all generate the same scalar potential $[15,18]$. In the absence of supersymmetry breaking, one can show that these classes can be related by the underlying noncompact $\frac{S U(2,1)}{S U(2) \times U(1)}$ no-scale symmetry [18]. Once the theory is coupled to matter and supersymmetry is broken, this symmetry is broken and different models will have different phenomenologies [26].

Neither of the superpotentials (3) nor (4) are responsible for supersymmetry breaking. In the absence of supersymmetry breaking, the minimum of the scalar potential is located at $\langle T\rangle=\frac{1}{2}$ and $\langle\phi\rangle=0$. Therefore, we need to extend our models and incorporate supersymmetry breaking. In the Cecotti model (3), supersymmetry can be broken by introducing a Polonyi field $z$ [74] with a superpotential,

$$
W_{\mathrm{P}}=\tilde{m}(z+b)
$$

\footnotetext{
${ }^{2}$ In this paper, we work in units of the reduced Planck mass $M_{P}=1 / \sqrt{8 \pi G_{N}} \simeq 2.4 \times 10^{18} \mathrm{GeV}$, unless explicitly noted.
}

where $b$ is a constant. It is important to note that the presence of a Polonyi field will shift the minimum, and in Sec. IV, we discuss this in more detail. If we consider the combined superpotential, $W=W_{C}+W_{P}$, we obtain an upper limit $\tilde{m}<m / 2$ for viable solutions with a Minkowski vacuum. The gravitino mass in this case is given by $m_{3 / 2}=\tilde{m} / \sqrt{3}$ and is lighter than the inflaton and can be a good dark matter candidate.

In the Wess-Zumino model (4), the superpotential is a function of a matterlike field $\phi$ only. In this case, one does not need to introduce an additional Polonyi field, and supersymmetry breaking occurs by introducing a constant $\lambda_{1}$ in the superpotential. More generally, we can add the following superpotential term $[19,20]$ :

$$
W_{\mathrm{SSB}}=\lambda_{1}-\lambda_{2}\left(2 T-\frac{\phi^{2}}{3}\right)^{3},
$$

which generates supersymmetry breaking through an $F$ term, which is given by $F_{T}=\lambda_{1}+\lambda_{2}$. In this case, the gravitino mass is given by $m_{3 / 2}=\lambda_{1}-\lambda_{2}$, and the vacuum energy density is expressed as $V_{0}=12 \lambda_{1} \lambda_{2}$ [75], which vanishes if either $\lambda_{1}$ or $\lambda_{2}$ is set to zero. For simplicity, we consider models with $\lambda_{2}=0$, and supersymmetry breaking is achieved by a constant $\lambda_{1}$, whose relative size is not restricted by the inflaton mass, $m$. We note that it is possible to add a linear term in the Wess-Zumino superpotential (4), which behaves as a Polonyi-like field [21]. However, this model has a strict upper bound on the gravitino mass of $m_{3 / 2} \lesssim 10^{6} \mathrm{GeV}$, and it is not valid for high-scale supersymmetry models. Another possibility is to introduce a Polonyi sector to the Wess-Zumino models [26]; however, in that case, the inflationary potential is affected, and the possibility for inflation becomes limited.

In both models, the phenomenological aspects for the limits $m_{3 / 2}>m$ and $m_{3 / 2}<m$ are distinct; therefore, we consider the four cases separately. The classification is shown in Table I.

It is crucial to note that for both types of models, stabilization of some fields is necessary and can be achieved dynamically by introducing higher-order correction terms in the Kähler potential. We consider the following general Kähler potential form:

$$
\begin{aligned}
K= & -3 \ln \left[T+\bar{T}+f(T, \bar{T})-\frac{|\phi|^{2}+|z|^{2}}{3}\right. \\
& +g(\phi, \bar{\phi})+h(z, \bar{z})]
\end{aligned}
$$

TABLE I. The classification of the high-scale supersymmetry models considered here.

\begin{tabular}{lll}
\hline \hline & $W_{\mathrm{C}}$ & $W_{\mathrm{WZ}}$ \\
\hline$m_{3 / 2}<m$ & $\mathrm{C}-1$ & $\mathrm{WZ}-1$ \\
$m_{3 / 2}>m$ & $\mathrm{C}-2$ & $\mathrm{WZ}-2$ \\
\hline \hline
\end{tabular}


where

$$
f(T, \bar{T})=0, \quad m g(\phi, \bar{\phi})=\frac{|\phi|^{4}}{\Lambda_{\phi}^{2}}, \quad h(z, \bar{z})=\frac{|z|^{4}}{\Lambda_{z}^{2}},
$$

for the Cecotti models, and

$$
\begin{aligned}
& f(T, \bar{T})=\frac{(T+\bar{T}-1)^{4}}{\Lambda_{T}^{2}}+\frac{d(T-\bar{T})^{4}}{\Lambda_{T}^{2}}, \\
& g(\phi, \bar{\phi})=0, \quad h(z, \bar{z})=0,
\end{aligned}
$$

for the Wess-Zumino models. A more detailed discussion related to stabilization can be found in [15,26,52,76-82]. For all correction terms, $\Lambda_{T}, \Lambda_{\phi}$, and $\Lambda_{z}$ are associated with the corresponding field stabilization, and they are assumed to be below the Planck scale $M_{P}$. As we discuss in the next section, due to supersymmetry breaking in Cecotti-type models, the vacuum expectation value (VEV) of a matterlike field $\phi$ is no longer zero, and to avoid the uplifting of Minkowski vacuum by strong stabilization effects, we impose the constraint $\langle\phi\rangle \lesssim \Lambda_{\phi}$.

One of the features of all the models discussed here is a high supersymmetry breaking scale. As noted earlier, we require that all sparticle masses are larger than the inflationary scale given by the inflaton mass with the possible exception of the gravitino. More specifically, we must (at least in some cases) generate a hierarchy between gaugino masses and the gravitino mass. Gaugino masses are given by

$$
m_{1 / 2}=\left|\frac{1}{2} e^{G / 2} \frac{\bar{f}_{z}}{\operatorname{Re} f}\left(G^{-1}\right)_{z}^{z} G^{z}\right| \simeq\left|\frac{1}{2} m_{3 / 2} \frac{\bar{f}_{z}}{\operatorname{Re} f}\right|,
$$

where $G=K+\log |W|^{2}$ is the Kähler function, $f_{\alpha \beta}=$ $f \delta_{\alpha \beta}$ is the gauge kinetic function, $f_{\alpha \beta} F_{\mu \nu}^{\alpha} F^{\beta \mu \nu}$. In the case of a strongly stabilized Polonyi field, we can write $f=f_{0}+f_{1} z / \Lambda_{z}$, where $f_{0} \sim 1 / g^{2}$ is related to the gauge coupling, and the VEV of $z$ is proportional to $\Lambda_{z}^{2} / M_{P} \ll$ $M_{P}$ (see below). Then $\bar{f}_{z} / f=f_{z} / f \sim g_{0}^{2} f_{1} / \Lambda_{z}$, and the gaugino mass is $m_{1 / 2} \sim g_{0}^{2} f_{1} m_{3 / 2} M_{P} / \Lambda_{z} \gg m_{3 / 2}$. Scalar masses may then receive contributions from gaugino loops so that $m_{0}^{2} \propto m_{1 / 2}^{2} / 16 \pi^{2}$ [27].

\section{MODELS OF LEPTOGENESIS}

Before we discuss leptogenesis in the context of the two inflationary paradigms, we first review some of the general formalism for generating a baryon asymmetry from a lepton asymmetry induced by the out-of-equilibrium decay of a heavy right-handed neutrino. For the most part, we concentrate on nonthermal leptogenesis [67]. We begin with the introduction of right-handed neutrinos and their role in the seesaw mechanism for generating neutrino masses. Later, we will associate one of the right-handed neutrinos with a fermionic partner of the inflaton [16]. We then give the basic formulae for generating a lepton asymmetry from the decays of right-handed neutrinos and its subsequent conversion to a baryon asymmetry through sphaleron interactions.

\section{A. The seesaw mechanism}

We begin our discussion by recalling the general features of the seesaw mechanism $[69,83]$. We introduce three generations of heavy right-handed neutrinos, that will produce a lepton asymmetry and generate the masses of the light neutrinos via the seesaw mechanism. In this case, the new terms in the Lagrangian are given by

$$
\mathcal{L} \supset-y_{i \alpha} \bar{N}_{i} L_{\alpha} H_{u}-\frac{1}{2} \bar{N}_{i}^{c} M_{i} N_{i}+\text { H.c. },
$$

where $\alpha=e, \mu, \tau, i=1,2,3$, and the Yukawa couplings are given by a $3 \times 3$ matrix $y$. For simplicity, we assume that the right-handed neutrino mass matrix $M$ is diagonal. ${ }^{3}$ To obtain the Dirac mass matrix via the seesaw mechanism, we use the following expression [69]:

$$
M_{\nu}=m_{D}^{T} M^{-1} m_{D},
$$

where $M^{-1}$ is the inverse of the diagonal right-handed neutrino mass matrix, and $m_{D}=y\left\langle H_{u}\right\rangle$, where $\left\langle H_{u}\right\rangle=$ $v \sin \beta$ with $v=174 \mathrm{GeV}$. The left-handed neutrino masses are obtained by diagonalizing the mass matrix $M_{\nu}$ (12) with the Pontecorvo-Maki-Nakagawa-Sakata (PMNS) matrix $U$,

$$
\operatorname{diag}\left\{m_{\nu_{1}}, m_{\nu_{2}}, m_{\nu_{3}}\right\}=U^{T} M_{\nu} U
$$

If we integrate out the heavy right-handed neutrinos, the left-handed neutrino masses become

$$
\left(M_{\nu}\right)_{\alpha \beta}=\sum_{i} y_{i \alpha} y_{i \beta} \frac{\left\langle H_{u}\right\rangle^{2}}{M_{i}} .
$$

For the consideration of nonthermal leptogenesis, we assume the following mass hierarchy: $2 M_{1} \lesssim m \ll M_{2}$, $M_{3}$, where $m \simeq 3 \times 10^{13} \mathrm{GeV}$ is the inflaton mass, so that lepton asymmetry will be primarily generated by the decays of the lightest right-handed neutrino $N_{1}{ }^{4}$

\footnotetext{
${ }^{3}$ Even if we do not assume that the right-handed neutrino matrix $M$ is diagonal, we can always diagonalize it by introducing a unitary matrix $U_{R}$.

${ }^{4}$ Even if $N_{2}$ and $N_{3}$ are also lighter than the inflaton and can be produced by inflaton decay, the following argument does not change drastically, as they generate the asymmetry in the same manner as $N_{1}$. Therefore, in the following argument, we assume that only $N_{1}$ among the three can be produced by the inflaton decay for the sake of simplicity.
} 
If we assume that the dominant contribution to the Yukawa matrix $y$ comes from the entry $y_{3 \tau} \equiv y_{3}$, the seesaw formula (14) leads to the following mass eigenvalue:

$$
m_{\nu_{3}} \simeq \frac{\left|y_{3}\right|^{2}\left\langle H_{u}\right\rangle^{2}}{M_{3}}
$$

which corresponds to the heaviest left-handed neutrino in the normal hierarchy. Thus, we have implicitly assumed that the neutrino mass matrix is nearly block diagonal (the flavor mixing terms are small) and that $\left|y_{3}\right|^{2} / M_{3}$ is the largest entry.

Analogously, we can consider the case when largest entry in the Yukawa matrix $y$ is $y_{2 \mu} \equiv y_{2}$, which leads to

$$
m_{\nu_{2}} \simeq \frac{\left|y_{2}\right|^{2}\left\langle H_{u}\right\rangle^{2}}{M_{2}}
$$

For a normal hierarchy $(\mathrm{NH})$ of the left-handed neutrinos, their masses are expressed as [84]

$$
m_{\nu_{2}} \simeq 0.0086 \mathrm{eV}, \quad m_{\nu 3} \simeq 0.0506 \mathrm{eV},
$$

and $m_{\nu_{1}}$ is very light. For inverted hierarchy $(I H)$, the neutrino masses are given by

$$
m_{\nu_{1}} \simeq 0.0497 \mathrm{eV}, \quad m_{\nu_{2}} \simeq 0.0504 \mathrm{eV},
$$

where $m_{\nu_{3}}$ is very light.

\section{B. Lepton asymmetry from heavy Majorana neutrino decays}

When the heavy right-handed Majorana neutrinos decay into leptons and Higgs bosons or their antiparticles, the lepton number is violated. The lepton asymmetry $\epsilon$ is generated by the interference between one-loop and tree diagrams of the following out-of-equilibrium decays of the lightest right-handed neutrino $N_{1}$ :

$$
\begin{aligned}
& N_{1} \rightarrow L_{\alpha}+H_{u} \\
& N_{1} \rightarrow \bar{L}_{\alpha}+\bar{H}_{u} .
\end{aligned}
$$

For our models of nonthermal leptogenesis, we assume $2 M_{1} \lesssim m \ll M_{2,3}$, where $m \simeq 3 \times 10^{13} \mathrm{GeV}$ is the mass of the inflaton. The expression for the $C P$ asymmetry is given by $[85,86]$

$$
\begin{aligned}
\epsilon & \equiv \frac{\Gamma_{N_{1} \rightarrow L_{\alpha} H_{u}}-\Gamma_{N_{1} \rightarrow \bar{L}_{\alpha} \bar{H}_{u}}}{\Gamma_{N_{1} \rightarrow L_{\alpha} H_{u}}+\Gamma_{N_{1} \rightarrow \bar{L}_{\alpha} \bar{H}_{u}}} \\
& \simeq \frac{1}{8 \pi} \frac{1}{\left(y y^{\dagger}\right)_{11}} \sum_{j=2,3} \operatorname{Im}\left(y y^{\dagger}\right)_{1 j}^{2} \cdot f\left(\frac{M_{j}^{2}}{M_{1}^{2}}\right),
\end{aligned}
$$

where

$$
f(x)=\sqrt{x}\left[\frac{1}{1-x}+1-(1+x) \ln \left(\frac{1+x}{x}\right)\right] .
$$

For $x \gg 1$, we use the approximation $f(x) \simeq-3 / 2 \sqrt{x}$, and the $C P$ asymmetry parameter (20) becomes

$\epsilon \simeq-\frac{3}{16 \pi} \frac{1}{\left(y y^{\dagger}\right)_{11}}\left[\operatorname{Im}\left(y y^{\dagger}\right)_{12}^{2} \frac{M_{1}}{M_{2}}+\operatorname{Im}\left(y y^{\dagger}\right)_{13}^{2} \frac{M_{1}}{M_{3}}\right]$.

If we consider the case when $y_{3 \tau}=y_{3}$ is significantly larger than other entries in the Yukawa matrix $y$ and $y_{1 \tau} \gg y_{1 e}$, $y_{1 \mu}$, we may neglect the $\operatorname{Im}\left(y y^{\dagger}\right)_{12}^{2}$ entry in (22) and write $\operatorname{Im}\left(y y^{\dagger}\right)_{13}^{2} \simeq\left|y_{1 \tau}\right|^{2}\left|y_{3 \tau}\right|^{2} \sin ^{2}(\theta-\phi)$ and $\left(y y^{\dagger}\right)_{11} \simeq\left|y_{1 \tau}\right|^{2}$, where we have introduced the phases $\theta$ and $\phi$ as $y_{1 \tau}=$ $\left|y_{1 \tau}\right| e^{i \theta}$ and $y_{3 \tau}=\left|y_{3 \tau}\right| e^{i \phi}$. Then, we define the effective $C P$-violating phase $\delta_{\text {eff }} \equiv \sin ^{2}(\theta-\phi)$, where $0 \leq \delta_{\text {eff }} \leq 1$, and express the $C P$ asymmetry parameter (22) as

$$
\epsilon \simeq-\frac{3 \delta_{\mathrm{eff}}\left|y_{3}^{2}\right|}{16 \pi} \frac{M_{1}}{M_{3}}
$$

and the $C P$-violating phase is given by

$$
\delta_{\mathrm{eff}}=\frac{1}{\left|y_{3}\right|^{2}} \frac{\operatorname{Im}\left(y y^{\dagger}\right)_{13}^{2}}{\left(y y^{\dagger}\right)_{11}} .
$$

Similarly, we can assume that $y_{2 \mu}=y_{2}$ is the largest entry in the Yukawa matrix $y$, and then $C P$ asymmetry parameter (22) becomes

$$
\epsilon \simeq-\frac{3 \delta_{\mathrm{eff}}\left|y_{2}^{2}\right|}{16 \pi} \frac{M_{1}}{M_{2}}
$$

If we then use the seesaw expression (15) or (16), we find

$$
\epsilon \simeq-\frac{3 \delta_{\mathrm{eff}}}{16 \pi} \cdot \frac{m_{\nu_{i}} M_{1}}{v^{2} \sin ^{2} \beta},
$$

where $i=2,3$ for normal hierarchy, and analogously, we can find the $C P$ asymmetry parameter (26) for inverted hierarchy, with $i=1,2$.

Equation (26) can be used to calculate the lepton asymmetry generated by the out-of-equilibrium decays of lightest right-handed neutrino $N_{1}$, and similar models were discussed in [87-91]. In the next section, we discuss how a lepton asymmetry is converted to a baryon asymmetry by sphaleron transitions.

\section{Production of baryon asymmetry}

We briefly discuss the mechanism which converts a lepton asymmetry into a baryon asymmetry via electroweak sphaleron interactions [70]. At high temperatures, the 
combination of a baryon and lepton number $B+L$ is violated, while the anomaly free parameter $B-L$ remains conserved. Sphaleron interactions are in equilibrium in the temperature range between $100 \mathrm{GeV}$ and $10^{12} \mathrm{GeV}$, and they convert a fraction of a nonzero $B-L$ asymmetry into a baryon asymmetry [71],

$$
Y_{B} \simeq a Y_{B-L},
$$

where $Y_{B}=n_{B} / s, Y_{B-L}=n_{B-L} / s$, and

$$
a=\frac{8 N_{F}+4 N_{H}}{22 N_{F}+13 N_{H}},
$$

where $N_{F}$ is the number of fermion generations and $N_{H}$ is the number of Higgs doublets. In our case, we have $N_{F}=3, N_{H}=1$, and $a=28 / 79$. In leptogenesis, where purely a lepton asymmetry is generated, $B-L=-L$.

For models of nonthermal leptogenesis, we impose the constraint $M_{1}>T_{\mathrm{RH}}{ }^{5}$ (the right-handed neutrino $N_{1}$ must be heavier than the reheating temperature $T_{\mathrm{RH}}$ ), and lepton asymmetry is produced through the out-of-equilibrium decay of the lightest right-handed neutrino $N_{1}$. In this case, we acquire the following expression for lepton asymmetry:

$$
Y_{L} \equiv \epsilon \frac{n_{N_{1}}}{s},
$$

and if we relate it to the baryon asymmetry number using Eq. (27), we find

$$
Y_{B} \simeq-a \epsilon \frac{n_{N_{1}}}{s} .
$$

If we combine the expressions (26) and (28) with (30), and assume that for high-scale supersymmetry models we have $\tan \beta \simeq 1$, we obtain the following expression for the baryon asymmetry:

$$
Y_{B} \simeq 7 \times 10^{-5} \delta_{\text {eff }} \frac{n_{N_{1}}}{s}\left(\frac{m_{\nu_{i}}}{0.05 \mathrm{eV}}\right)\left(\frac{M_{1}}{10^{12} \mathrm{GeV}}\right),
$$

where $i=2,3$.

Finally, the baryon asymmetry of the Universe is given by the most recent Planck data constraints [13],

$$
\eta_{B}=\frac{n_{B}-n_{\bar{B}}}{n_{\gamma}} \simeq 6.12 \times 10^{-10}, \quad Y_{B} \simeq 8.7 \times 10^{-11} .
$$

\footnotetext{
${ }^{5}$ To preserve the lepton asymmetry, we require that the lepton number violating interaction, which is characterized by an operator $y^{2} L L H_{u} H_{u} / M$, remains out-of-equilibrium when sphaleron transitions are in thermal equilibrium. It was shown in [92], that for high-scale supersymmetry models, we must satisfy the constraint $M /|y|^{2}>10^{13.5}$, or $m_{\nu}<0.5 \mathrm{eV}$.
}

\section{CECOTTI-TYPE MODELS OF INFLATION}

We begin by considering the inflationary models where the inflaton is associated with the volume modulus $T$. In particular, we consider the superpotential form $W_{C}$, given by Eq. (3). However, for Cecotti-type models, one cannot introduce a constant term in the superpotential, because it shifts the original minimum to a new supersymmetry preserving the anti-de Sitter (AdS) vacuum [20,26]. Therefore, we introduce a Polonyi sector and consider the Kähler potential form (7) with the superpotential $W_{C}+W_{P}$, where $W_{P}$ is given by Eq. (5).

The addition of a Polonyi sector shifts the scalar potential minimum to a new vacuum with broken supersymmetry. In the absence of superpotential $W_{C}$, the strongly stabilized Polonyi potential has a minimum at $\langle z\rangle \simeq \Lambda_{z}^{2} / 2 \sqrt{3}$, where we have omitted the higher-order terms in $\Lambda_{z}$. If we choose a constant $b \simeq 1 / \sqrt{3}$, we obtain a vanishing vacuum energy density $V=0$.

When we consider the superpotential combination $W_{C}+W_{P}$, the VEVs of the fields $T, \phi$, and $z$ shift. However, the VEVs of the shifted fields will depend on whether the parameter $\Delta \equiv \tilde{m} / m>1 / 2$ or $\Delta<1 / 2$. For the latter case, the supersymmetry breaking Minkowski minimum $V=0$ is located at

$$
\begin{gathered}
\langle T\rangle=\frac{1}{6}\left(4-\sqrt{1-4 \Delta^{2}}\right), \\
\langle\phi\rangle= \pm\left(\frac{1}{2}-\frac{1}{2} \sqrt{1-4 \Delta^{2}}\right)^{1 / 2}, \\
\langle z\rangle=\mp \frac{\Lambda_{z}^{2}}{6 \sqrt{6} \Delta}\left(1-\sqrt{1-4 \Delta^{2}}\right)^{1 / 2} \\
b= \pm \frac{\sqrt{6}}{18 \Delta}\left(2+\sqrt{1-4 \Delta^{2}}\right)\left(1-\sqrt{1-4 \Delta^{2}}\right)^{1 / 2},
\end{gathered}
$$

where the higher-order terms in $\Lambda_{z}$ have been omitted. For small values of $\Delta$, the VEVs (33a)-(33d) can be expanded to

$$
\begin{array}{rlrl}
\langle T\rangle & \simeq \frac{1}{2}+\frac{\Delta^{2}}{3}, & \langle\phi\rangle \simeq \pm \Delta, \\
\langle z\rangle \simeq \pm \frac{\Lambda_{z}^{2}}{6 \sqrt{3}}, & b \simeq \pm \frac{1}{\sqrt{3}} \mp \frac{\Delta^{2}}{6 \sqrt{3}},
\end{array}
$$

which agrees with the previous results [26,27]. One can see from Eqs. (33a)-(33d) that the largest possible value is $\Delta=1 / 2$, and larger values of the parameter $\Delta$ lead to a positive vacuum energy density of order $\tilde{m}^{2} \Lambda_{z}^{2}$. However, if we modify the Cecotti superpotential (3), it is possible to accommodate the values $\Delta>1 / 2$, and we discuss this possibility in Sec. IV B. 
Using the vacuum expectation values (33a)-(33d), we consider two separate Cecotti-type models: C-1 models, where the gravitino is lighter than the inflaton, $m_{3 / 2}<m$, and C-2 models, where the gravitino is heavier than the inflaton, $m_{3 / 2}>m$.

\section{A. C-1 models}

For C-1 models, the gravitino plays the role of the dark matter candidate, and all other sparticles are taken to be heavier than the inflaton field [27]. It was shown in the previous section that $\mathrm{C}-1$ models are valid when $\Delta=$ $\sqrt{3} m_{3 / 2} / m<1 / 2$, or $m_{3 / 2}<m / 2 \sqrt{3}$, which shows that the gravitino is lighter than the inflaton.

We begin by considering the relevant supergravity Lagrangian for the scalar fields,

$$
\mathcal{L}=-G_{i \bar{j}} \partial_{\mu} \phi^{i} \partial^{\mu} \phi^{\bar{j}}-V,
$$

where $V$ is the effective scalar potential generated by $F$ term contributions, and we defined the Kähler function as $G=K+\ln W+\ln \bar{W}$. From the Kähler potential form (7) and the Lagrangian (35), we find that the canonically normalized fields are expressed as

$$
\begin{gathered}
T=\frac{1}{2}\left(e^{\left.\sqrt{\frac{2}{3} T_{R}}+i \sqrt{\frac{2}{3}} T_{I}\right),}\right. \\
\phi=\frac{1}{\sqrt{2}}\left(\phi_{R}+i \phi_{I}\right), \\
z=\frac{1}{\sqrt{2}}\left(z_{R}+i z_{I}\right),
\end{gathered}
$$

where $T_{R}$ is the inflaton. Then, if we assume that $\Delta \ll 1$ and neglect the higher-order contributions, we obtain the following masses for the canonically normalized fields:

$$
\begin{aligned}
m_{S_{1 R}}^{2} \simeq\left(1+\frac{\Delta}{\sqrt{3}}\right) m^{2}, & m_{S_{1 R}} \simeq\left(1+\frac{\Delta}{2 \sqrt{3}}\right) m, \\
m_{S_{2 R}}^{2} \simeq\left(1-\frac{\Delta}{\sqrt{3}}\right) m^{2}, & m_{S_{2 R}} \simeq\left(1-\frac{\Delta}{2 \sqrt{3}}\right) m, \\
m_{S_{1 I}}^{2} \simeq\left(1-\frac{\Delta}{\sqrt{3}}\right) m^{2}, & m_{S_{1 I}} \simeq\left(1-\frac{\Delta}{2 \sqrt{3}}\right) m, \\
m_{S_{2 I}}^{2} \simeq\left(1+\frac{\Delta}{\sqrt{3}}\right) m^{2}, & m_{S_{2 I}} \simeq\left(1+\frac{\Delta}{2 \sqrt{3}}\right) m, \\
m_{z_{R}}^{2} \simeq 12 m^{2} \frac{\Delta^{2}}{\Lambda_{z}^{2}}, & m_{z_{R}} \simeq 2 \sqrt{3} m \frac{\Delta}{\Lambda_{z}},
\end{aligned}
$$

$$
\begin{gathered}
m_{z_{I}}^{2} \simeq 12 m^{2} \frac{\Delta^{2}}{\Lambda_{z}^{2}}, \quad m_{z_{I}} \simeq 2 \sqrt{3} m \frac{\Delta}{\Lambda_{z}}, \\
m_{3 / 2} \simeq m \frac{\Delta}{\sqrt{3}},
\end{gathered}
$$

where the eigenstates $S_{1,2 R}$ correspond to equal mixtures of the real states $T_{R}$ and $\phi_{R}$, and the eigenstates $S_{1,2 I}$ correspond to equal mixtures of the imaginary states $T_{I}$ and $\phi_{I}$, given by

$$
\begin{aligned}
S_{1 R} & \simeq \frac{1}{\sqrt{2}}\left(T_{R}-\phi_{R}\right), & S_{2 R} & \simeq \frac{1}{\sqrt{2}}\left(T_{R}+\phi_{R}\right), \\
S_{1 I} & \simeq \frac{1}{\sqrt{2}}\left(T_{I}-\phi_{I}\right), & S_{2 I} & \simeq \frac{1}{\sqrt{2}}\left(T_{I}+\phi_{I}\right) .
\end{aligned}
$$

It is important to note that the Polonyi field mixing with fields $T_{R, I}$ and $\phi_{R, I}$ was neglected. In order to ensure that the entropy production from the Polonyi sector is sufficiently small [82], and to avoid the production of the particles $z_{R, I}$ from the inflaton decays, we assume that $2 \sqrt{3} \Delta \lesssim \Lambda_{z} \lesssim 10^{-2}$.

Next, we consider the Lagrangian terms for the lefthanded chiral fermions $\chi_{L}$, given by

$$
\mathcal{L} \supset-g_{i j} \bar{\chi}_{L}^{i} D \chi_{L}^{\bar{j}}-\left(\frac{1}{2} m_{i j} \bar{\chi}_{L}^{i} \chi_{L}^{j}+\text { H.c. }\right),
$$

with

$$
\begin{gathered}
g_{i \bar{j}}=G_{i \bar{j}}-\frac{1}{3} G_{i} G_{\bar{j}}, \\
m_{i j}=G_{i j}+\frac{1}{3} G_{i} G_{j}-\Gamma_{i j}^{k} G_{k},
\end{gathered}
$$

where we subtracted the Goldstino mode. Here, we defined, $G_{i}=\partial G / \partial \phi^{i}$ and $G_{i j}=\partial^{2} G / \partial \phi^{i} \partial \phi^{j}$, where $\phi^{i}$ is the scalar partner of $\chi_{L}^{i}$, and $\Gamma_{i j}^{k}$ are the Christoffel symbols (for a more detailed discussion, see [93]). It should be noted that in general $g_{i \bar{j}}$ is not the identity matrix, and relevant fields should be canonically normalized. In our case, the Goldstino is identified with the fermionic partner of the Polonyi field $z$, and the physical masses of the remaining fermions are given by

$$
\begin{aligned}
& m_{\chi_{1}} \simeq\left(1+\frac{\Delta \Lambda_{z}^{2}}{18 \sqrt{3}}\right) m, \\
& m_{\chi_{2}} \simeq\left(1-\frac{\Delta \Lambda_{z}^{2}}{18 \sqrt{3}}\right) m .
\end{aligned}
$$

As in the scalar field case, the fermion mass eigenstates are a mixture of eigenstates $\chi_{T}$ and $\chi_{\phi}$, which are related to $\chi_{1}$ and $\chi_{2}$ by 


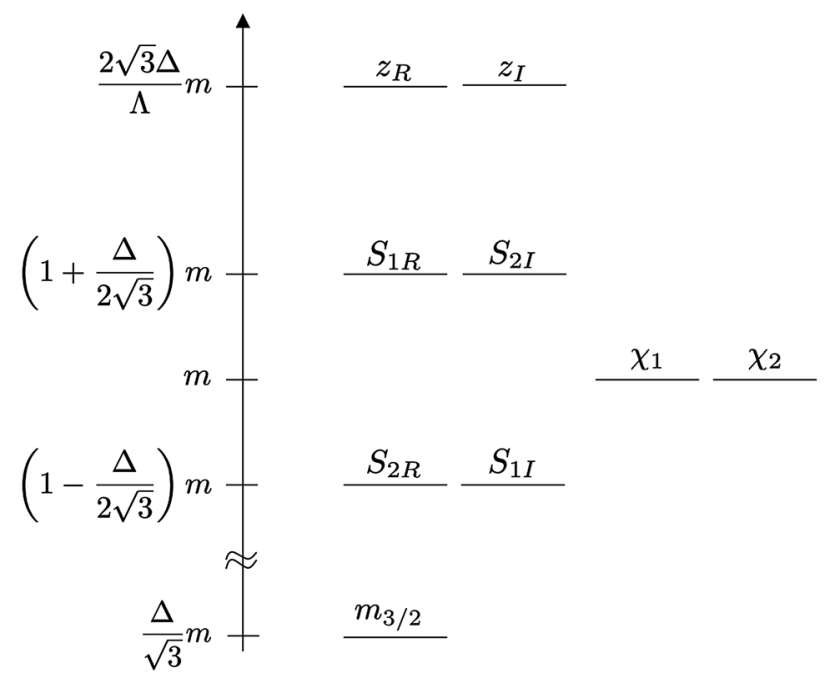

FIG. 1. Mass spectrum of model C-1.

$$
\chi_{1} \simeq \frac{1}{\sqrt{2}}\left(\chi_{T}+\chi_{\phi}\right), \quad \chi_{2} \simeq \frac{1}{\sqrt{2}}\left(\chi_{T}-\chi_{\phi}\right) .
$$

Although the states $S_{1 R}$ and $S_{2 I}$ are heavier than the scalar states $S_{2 R}$ and $S_{1 I}$ or the fermion states $\chi_{1,2}$, the decays to lighter states are kinematically forbidden. For example, if we would consider the decay channel of the state $S_{1 R}$ into a fermion $\chi_{1,2}$ and a gravitino, the mass splitting of the states is smaller than the gravitino mass, i.e., $m_{S_{1 R}}-m_{\chi_{1,2}} \simeq m_{3 / 2} / 2$, and the decay is kinematically forbidden $[38,94]$. Finally, we show the mass spectrum for model C-1 in Fig. 1.

\section{B. C-2 models}

For C-2 models, the inflaton decay does not produce gravitinos because the gravitino mass is heavier than the inflaton, $m_{3 / 2}>m$, and we need to consider a different dark matter candidate. In this paper, we mostly focus on inflation and leptogenesis, and studies related to different dark matter candidates are left for future work.

If we look at the VEVs of fields $T, \phi$, and $z$, given by Eqs. (33a)-(33d), we see that viable models with Minkowski vacua impose the constraint $\Delta<1 / 2$. However, this constraint can be avoided if we modify the superpotential (3). For example, consider the superpotential,

$$
W_{C}=\sqrt{3} m \phi\left(T-\frac{1}{2}+g \phi^{2}\right),
$$

where we introduced the term $g \phi^{2}$. Because we dynamically stabilize a matterlike field to $\phi=0$ during inflation, the additional term in (48) does not affect the inflationary potential. The introduction of a new term alters the solutions for the field VEVs, which become

$$
\begin{gathered}
\langle T\rangle=\frac{4-63 g+(9 g-1) \sqrt{1-4 \Delta^{2}(1-18 g)}}{6-108 g}, \quad \text { (49a) } \\
\langle\phi\rangle= \pm \sqrt{\frac{1-\sqrt{1-4 \Delta^{2}(1-18 g)}}{2(1-18 g)}}, \\
\langle z\rangle= \pm \frac{\Lambda^{2} \sqrt{1-\sqrt{1-4 \Delta^{2}(1-18 g)}}}{6 \sqrt{6} \Delta \sqrt{1-18 g}}, \\
b= \pm \frac{\sqrt{1-\sqrt{1-4 \Delta^{2}(1-18 g)}\left(2+\sqrt{1-4 \Delta^{2}(1-18 g)}\right)}}{3 \sqrt{6} \Delta \sqrt{1-18 g}} .
\end{gathered}
$$

In the limit, $g \rightarrow 0$, we recover the solutions (33a)-(33d), given for the model C-1. However, when $\Delta>1 / 2$, we see from Eqs. (49a)-(49d), that in order to maintain the real values for the shifted VEVs, we must satisfy the following constraints on $g$ :

$$
1-18 g \geq 0, \quad \sqrt{1-4 \Delta^{2}(1-18 g)} \geq 0 .
$$

We find the following inequality for the constant $g$ :

$$
g \geq \frac{1}{18}-\frac{1}{72 \Delta^{2}}
$$

which for large values of $\Delta$ can be approximated to $g \simeq 1 / 18$. In most cases to obtain a viable solution with a Minkowski vacuum at the minimum, we will need to choose a value of $g$, which is very close to the upper bound $g \simeq 1 / 18$; otherwise, the potential is uplifted resulting in a positive vacuum energy density.

To obtain a viable model with $\Delta>1 / 2$, we need to introduce a shift in the stability correction in the Kähler potential,

$K=-3 \ln \left(T+\bar{T}-\frac{|\phi|^{2}}{3}-\frac{|z|^{2}}{3}+\frac{|z|^{4}}{\Lambda_{z}^{2}}+\frac{|\phi-\Delta|^{4}}{\Lambda_{\phi}^{2}}\right)$,

where the dynamical stabilization now occurs around the shifted VEV of $\langle\phi\rangle=\Delta$ rather than about $\langle\phi\rangle=0$. However, due to complexity of the model, it cannot be solved analytically, and we analyze it numerically. We consider a concrete example with $\Delta=2$, and choose the following parameters for our numerical study:

$$
g=0.05315 \simeq \frac{1}{18}, \quad \Lambda_{z}^{2}=\Lambda_{\phi}^{2}=0.1 .
$$

If we use Eqs. (49a)-(49d) with (53), we find

$$
\langle T\rangle \simeq 1.20, \quad\langle\phi\rangle \simeq 2, \quad\langle z\rangle \simeq 0.11, \quad b \simeq 0.56 .
$$




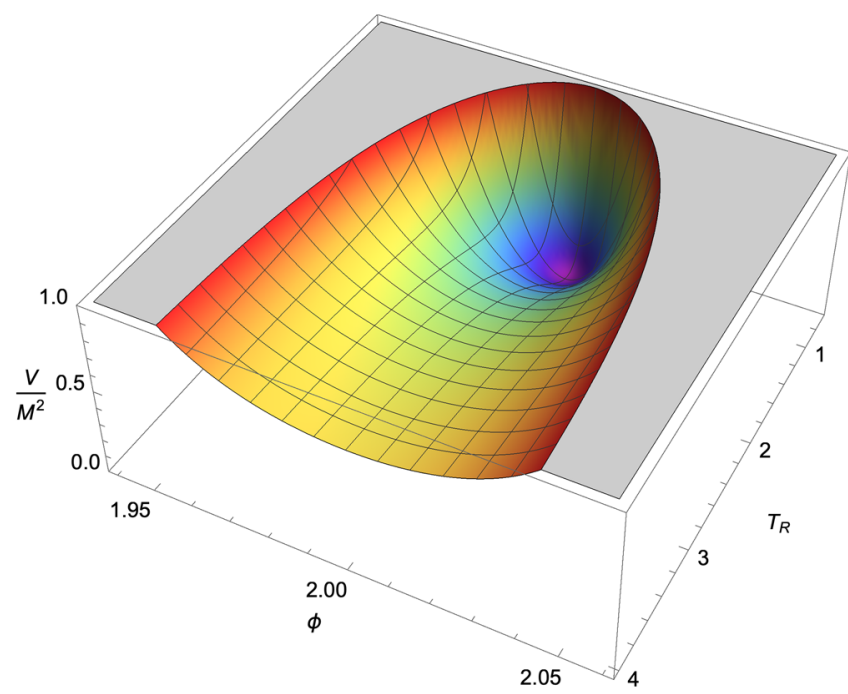

FIG. 2. Realization of the Starobinsky-like inflationary potential in the model C-2 for $\Delta=2$. The minimum of the potential is located at $\left\langle T_{R}\right\rangle \simeq 1.07$ and $\langle\phi\rangle \simeq 2$.

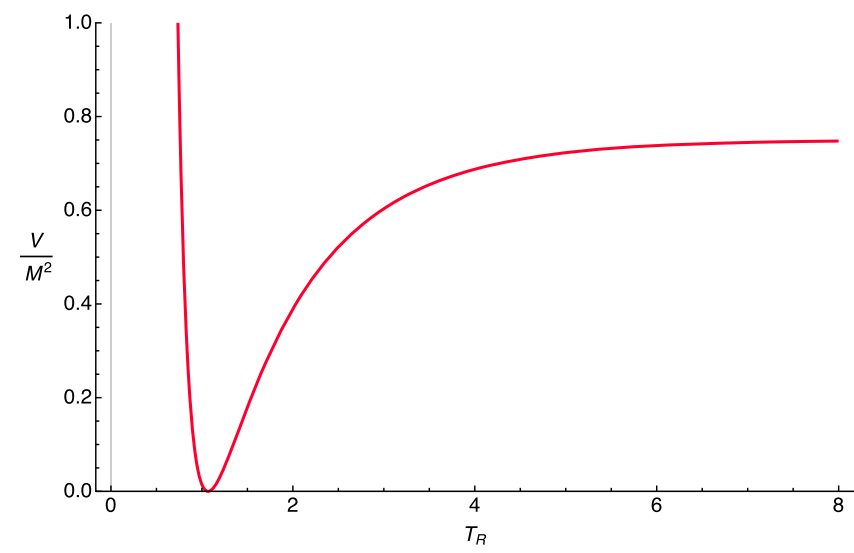

FIG. 3. Realization of the Starobinsky-like inflationary potential in the model C-2 for $\Delta=2$ when a matterlike field is fixed to $\langle\phi\rangle \simeq 2$.

To find the canonically normalized field $T_{R}$, which drives inflation, we use Eq. (36), and we find that the VEV of canonically normalized field is given by $\left\langle T_{R}\right\rangle \simeq 1.07$. In Figs. 2 and 3, we plot the Starobinsky-like inflationary potential for a case corresponding to $\mathrm{C}-2$ with $\Delta=2$. Figure 2 shows the effective scalar potential $V$ as a function of fields $T_{R}$ and $\phi$. The Starobinsky-like inflationary plot with fixed value of $\langle\phi\rangle \simeq 2$ is shown in Fig. 3 .

Next, we find the relevant scalar and fermion masses for our particular example. The scalar masses are given by

$$
\begin{aligned}
& m_{T_{R}} \simeq m, \\
& m_{T_{I}} \simeq m,
\end{aligned}
$$

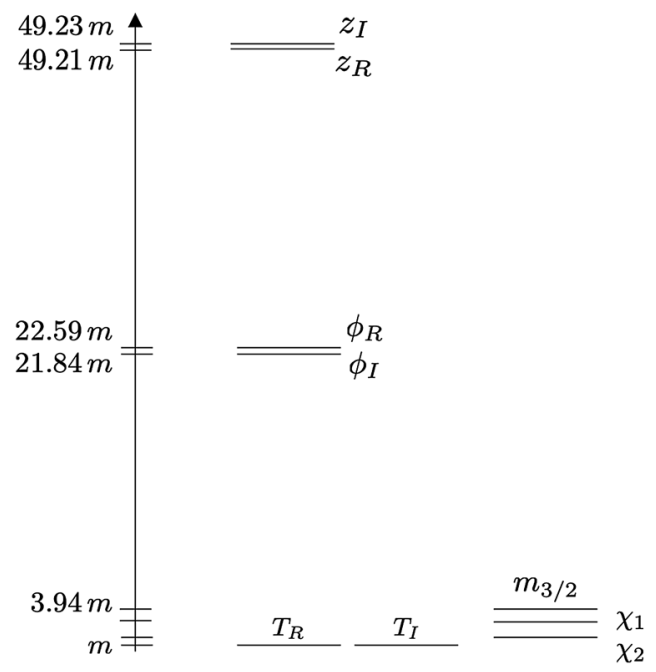

FIG. 4. Mass spectrum of the model C-2. In this scenario, $T_{R}$ is identified as the inflaton, and $m_{T_{R}} \simeq m<m_{3 / 2}$.

$$
\begin{aligned}
& m_{\phi_{R}} \simeq 22.59 m, \\
& m_{\phi_{I}} \simeq 21.84 m, \\
& m_{z_{R}} \simeq 49.21 \mathrm{~m}, \\
& m_{z_{I}} \simeq 49.23 \mathrm{~m},
\end{aligned}
$$

where, in this case, we no longer have maximal mixing between the fields $T_{R}$ and $\phi_{R}$ or $T_{I}$ and $\phi_{I}$. If we eliminate the Goldstino mode from the spectrum, which, in this case, is a mixture of the fermion fields $\chi_{T}, \chi_{\phi}$, and $\chi_{z}$, given by

$$
\eta \simeq-2.03 \chi_{T}+2.43 \chi_{\phi}+0.48 \chi_{z},
$$

we find the following fermion masses:

$$
\begin{aligned}
& m_{\chi_{1}}=2.82 m, \\
& m_{\chi_{2}}=1.39 m .
\end{aligned}
$$

Because of the substantial shifts in the VEVs given in Eqs. (49a)-(49d) due to large $\Delta$, we can no longer simply approximate $m_{3 / 2}=m \Delta / \sqrt{3}$. Instead, we find numerically that the gravitino mass is given by

$$
m_{3 / 2} \simeq 3.94 m,
$$

where as expected for C-2 models, we have $m_{3 / 2}>m$.

Finally, we show the mass spectrum for this particular example of the model C-2 in Fig. 4. 


\section{LEPTOGENESIS IN CECOTTI-TYPE MODELS}

In this section, we study Cecotti-type models of nonthermal leptogenesis for high-scale supersymmetry. Despite the differences in their spectra, leptogenesis in both types of models, C-1 and C-2, is the same. Of course, in $\mathrm{C}-1$, the gravitino may be the dark matter produced during reheating or direct decays, while in $\mathrm{C}-2$, an additional dark matter candidate must be introduced.

For Cecotti-type models, reheating proceeds via the gravitational coupling of the inflaton $T_{R}$ to Higgs bosons, and its decay rate is given by $[26,27,55,56]$

$$
\Gamma_{2 h}=\frac{\mu^{4}}{12 \pi m M_{P}^{2}} \equiv \frac{\lambda^{2}}{8 \pi} m,
$$

where we define a Yukawa-like coupling $\lambda \equiv \sqrt{\frac{2}{3} \frac{\mu^{2}}{m M_{P}}}$. We also took into account the fact that for high-scale supersymmetry models, the Higgs boson has 4 degrees of freedom. The reheating temperature is expressed as [27]

$$
T_{\mathrm{RH}} \simeq\left(\frac{40}{g_{*} \pi^{2}}\right)\left(\frac{\Gamma_{2 h} M_{P}}{c}\right)^{1 / 2},
$$

where $g_{*}=427 / 4$ is the effective degrees of freedom of the Standard model, and $c \simeq 1.2$. We can also express the reheating temperature (61) in terms of the coupling $\lambda$ as $T_{\mathrm{RH}} \simeq 0.5(\lambda / 2 \pi) \sqrt{m M_{P}}$, and the maximal temperature attained during reheating is given by $T_{\max } \simeq 0.5\left(8 \pi / \lambda^{2}\right)^{1 / 4} T_{\mathrm{RH}}$.

It was shown in $[27,56]$ that to obtain the correct dark matter relic density, we require the $\mu$ term to be in the range of $m \lesssim \mu \simeq 3 \times 10^{13}-10^{15} \mathrm{GeV}$, which we also expect from the fact the Higgsino mass parameter should lie above the inflaton mass in high-scale supersymmetry models. We then express the reheating temperature (61) as

$T_{\mathrm{RH}} \simeq 7.73 \times 10^{10} \mathrm{GeV}\left(\frac{\mu}{10^{14} \mathrm{GeV}}\right)^{2}\left(\frac{m}{3 \times 10^{13} \mathrm{GeV}}\right)^{-1 / 2}$.

The relic density of gravitinos is dependent on the reheat temperature and, through Eq. (62), on $\mu$. As noted earlier, there are two contributions to the gravitino relic density. It is produced thermally by the annihilations of Standard Model particles and directly through inflaton decays. The annihilations depend on $T_{\mathrm{RH}}^{7}[27,54-56,63]$ and hence, on $\mu^{14}$. Inflaton decays may also produce a sizeable contribution to the gravitino density. Tree level decays are suppressed for small $\Lambda_{z}$, but decays through Higgs loops are always present and in fact, dominate over the annihilations when $m_{3 / 2} \lesssim 0.1 m$ [56]. The gravitino abundance through decays depends linearly on $T_{\mathrm{RH}} \propto \mu^{2}$, which has also a weak (logarithmic) dependence of the branching ratio on $\mu$ [56].

In the case of nonthermal leptogenesis, we must satisfy the constraint $T_{\mathrm{RH}} \lesssim M_{1}$, and if we assume the lower bound of the $\mu$ term, given by $\mu \simeq m \simeq 3 \times 10^{13} \mathrm{GeV}$, we obtain

$$
M_{1} \gtrsim 7 \times 10^{9} \mathrm{GeV} .
$$

We begin our analysis by considering the following addition to the superpotential, which characterizes the seesaw mechanism and leptogenesis in Cecotti-type models:

$$
W \supset y_{i \alpha} N_{i} L_{\alpha} H_{u}+\frac{1}{2} N_{i} M_{i} N_{i},
$$

where $i=1,2,3, \alpha=e, \mu, \tau$, and $y$ is the Yukawa coupling matrix, where for simplicity, we have assumed that the righthanded neutrino mass matrix $M$ is diagonal. We assume the mass hierarchy $2 M_{1}<m \ll M_{2}, M_{3}$, and in all cases that we consider, the decays of the lightest right-handed neutrino $N_{1}$ will be responsible for the dominant contribution to the generation of the lepton asymmetry.

Next, we also consider the two-body decay channel of the inflaton to the lightest of the right-handed neutrinos, which can be calculated from the superpotential (64), and is given by [26]

$$
\Gamma_{2 N_{1}}=\frac{M_{1}^{2} m}{192 \pi M_{p}^{2}}\left(1-\frac{4 M_{1}^{2}}{m^{2}}\right)^{3 / 2},
$$

where we included the kinetic factor $\left(1-4 M_{1}^{2} / m^{2}\right)^{3 / 2}$ to account for cases when $2 M_{1} \lesssim m$. Most importantly, this decay channel will be responsible for the nonthermal production of the lightest right-handed neutrinos, which then decay into leptons and Higgs bosons, and produce a lepton asymmetry. Because our inflaton mass is $m \simeq 3 \times$ $10^{13} \mathrm{GeV}$ and $\mu \gtrsim m$, the decay channel to Higgs bosons is the dominant channel, and $\Gamma_{2 h} \gg \Gamma_{2 N_{1}}$. The branching ratio of the two decay channels is given by

$$
B_{R}=\frac{\Gamma_{2 N_{1}}}{\Gamma_{2 h}} \simeq \frac{M_{1}^{2} m^{2}}{16 \mu^{4}}\left(1-\frac{4 M_{1}^{2}}{m^{2}}\right)^{3 / 2} \lesssim 10^{-3} .
$$

It is important to note that we assume that the lightest righthanded neutrino $N_{1}$ decays instantaneously to leptons and Higgs bosons. As such, we must require $\Gamma_{L_{\alpha} h}>\Gamma_{2 N_{1}}$, which will be justified at the end of this section.

In order to obtain the number density of the lightest right-handed neutrinos $n_{N_{1}}$, we assume noninstantaneous reheating. In this case, we find [56]

$$
n_{N_{1}}\left(T_{R H}\right)=\frac{g_{*} \pi^{2}}{18 m} T_{R H}^{4} N B_{R},
$$

where $N$ is the number of the lightest right-handed neutrinos $N_{1}$ produced by the inflaton $T_{R}$ decay, which 


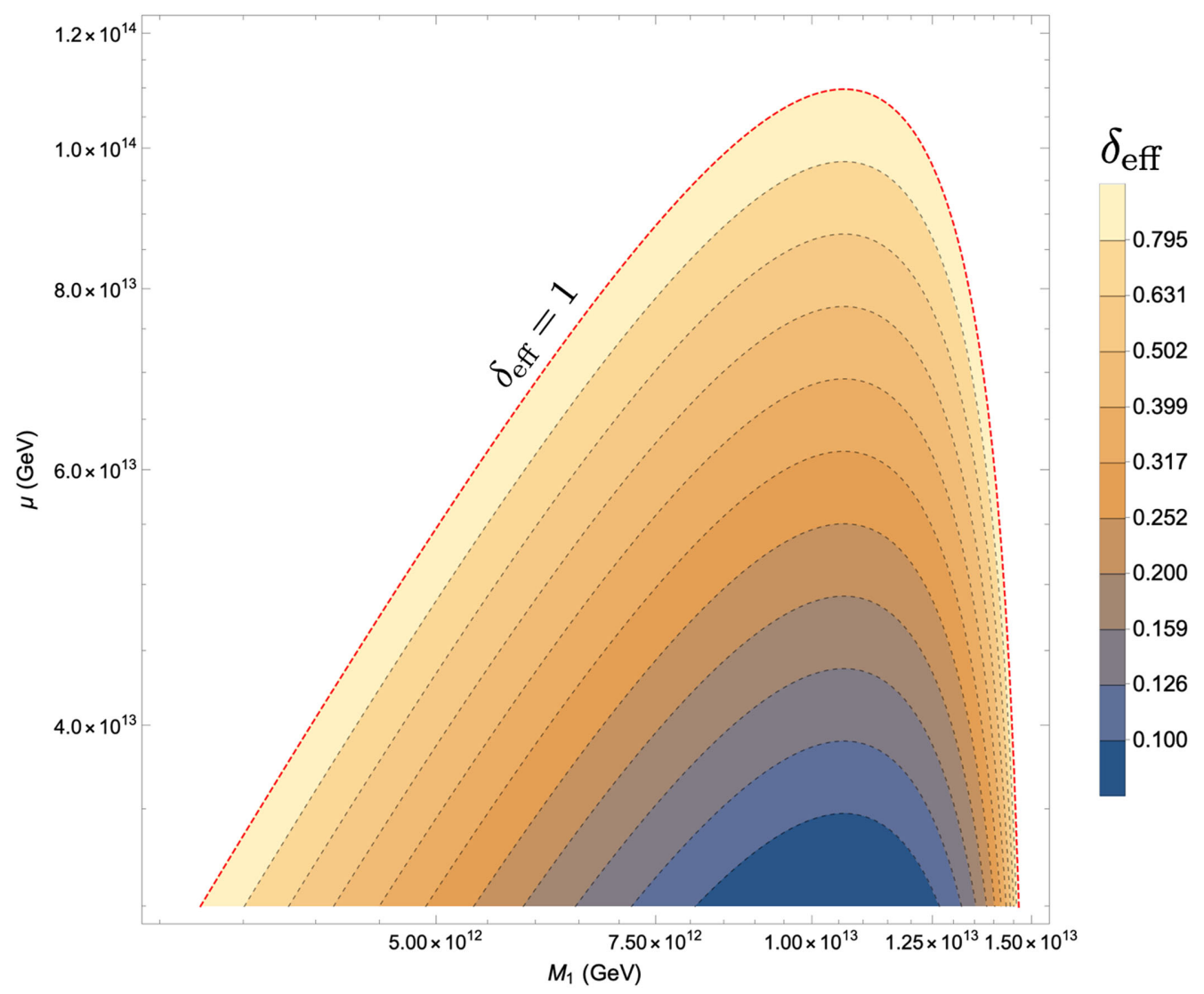

FIG. 5. Range of right-handed neutrino masses $M_{1}$ and the $\mu$ term satisfying the baryon asymmetry $Y_{B} \simeq 8.7 \times 10^{-11}$, with $m_{\nu_{3}} \simeq$ $0.05 \mathrm{eV}$ in the models $\mathrm{C}-1$ or $\mathrm{C}-2$. The red-dashed line corresponds to limit of maximal $C P$-violating phase $\delta_{\text {eff }}$.

is $N=2$ in our case, and $B_{R}$ is the branching ratio of the inflaton to right-handed neutrino decay, given by (66). The ratio of the number density of $N_{1}$ to entropy is

$$
\frac{n_{N_{1}}}{s} \simeq \frac{5 m M_{1}^{2} T_{\mathrm{RH}}}{32 \mu^{4}}\left(1-\frac{4 M_{1}^{2}}{m^{2}}\right)^{3 / 2}
$$

where the entropy density is $s=\frac{2 \pi^{2}}{45} g_{*} T^{3}$.

To obtain the baryon asymmetry, we can use Eqs. (68) and (31) and find

$$
\begin{aligned}
Y_{B} \simeq & 2.5 \times 10^{-13} \delta_{\mathrm{eff}}\left(\frac{\mu}{10^{14} \mathrm{GeV}}\right)^{-2}\left(\frac{m_{\nu_{i}}}{0.05 \mathrm{eV}}\right) \\
& \times\left(\frac{M_{1}}{10^{12} \mathrm{GeV}}\right)^{3}\left(\frac{m}{3 \times 10^{13} \mathrm{GeV}}\right)^{1 / 2}\left(1-\frac{4 M_{1}^{2}}{m^{2}}\right)^{3 / 2},
\end{aligned}
$$

where $i=2,3$ for the normal hierarchy given by Eq. (17), and $i=1,2$ for the inverse hierarchy given by Eq. (18). Therefore, if we use the observationally determined value for the baryon asymmetry $Y_{B} \simeq 8.7 \times 10^{-11}$, we obtain the following constraint:

$$
\begin{gathered}
\delta_{\mathrm{eff}}^{1 / 3}\left(\frac{\mu}{10^{14} \mathrm{GeV}}\right)^{-2 / 3}\left(\frac{m_{\nu_{i}}}{0.05 \mathrm{eV}}\right)^{1 / 3}\left(\frac{M_{1}}{10^{12} \mathrm{GeV}}\right) \\
\quad \times\left(\frac{m}{3 \times 10^{13} \mathrm{GeV}}\right)^{1 / 6}\left(1-\frac{4 M_{1}^{2}}{m^{2}}\right)^{1 / 2} \simeq 7
\end{gathered}
$$

This constraint is used in Fig. 5 to find the allowed values $\mu$, $M_{1}$, and $\delta_{\text {eff }}$, that can accommodate the observed value of baryon asymmetry $Y_{B}$ for fixed $m=3 \times 10^{13} \mathrm{GeV}$ and $m_{\nu_{3}} \simeq 0.05 \mathrm{eV}$. For each pair of points $\left(M_{1}, \mu\right)$, the shading corresponds to the required value of $\delta_{\text {eff }}$ to obtain the correct baryon asymmetry. An analogous plot using $m_{\nu_{2}} \simeq$ $0.0086 \mathrm{eV}$ is shown in Fig. 6.

We see from these figures that there is a maximum value of $\mu \lesssim 1.1 \times 10^{14} \mathrm{GeV}\left(4.6 \times 10^{13} \mathrm{GeV}\right)$ using $m_{\nu_{3}}\left(m_{\nu_{2}}\right)$ in Eq. (70). It is rather amazing that the range of $\mu$ required to obtain the correct baryon asymmetry coincides with the value of $\mu$ needed to obtain the correct relic density of gravitino dark matter in $\mathrm{C}-1$ type models (recall, there is no gravitino dark matter in $\mathrm{C}-2$ models).

In the case of the inverted hierarchy of the left-handed neutrinos, using Eq. (18), the limits in the $\left(M_{1}, \mu, \delta_{\text {eff }}\right)$ parameter space are very similar to the results shown in Fig. 5 because $I H$ neutrino masses are very close to $m_{\nu_{3}} \simeq$ $0.05 \mathrm{eV}$ in the $N H$. 


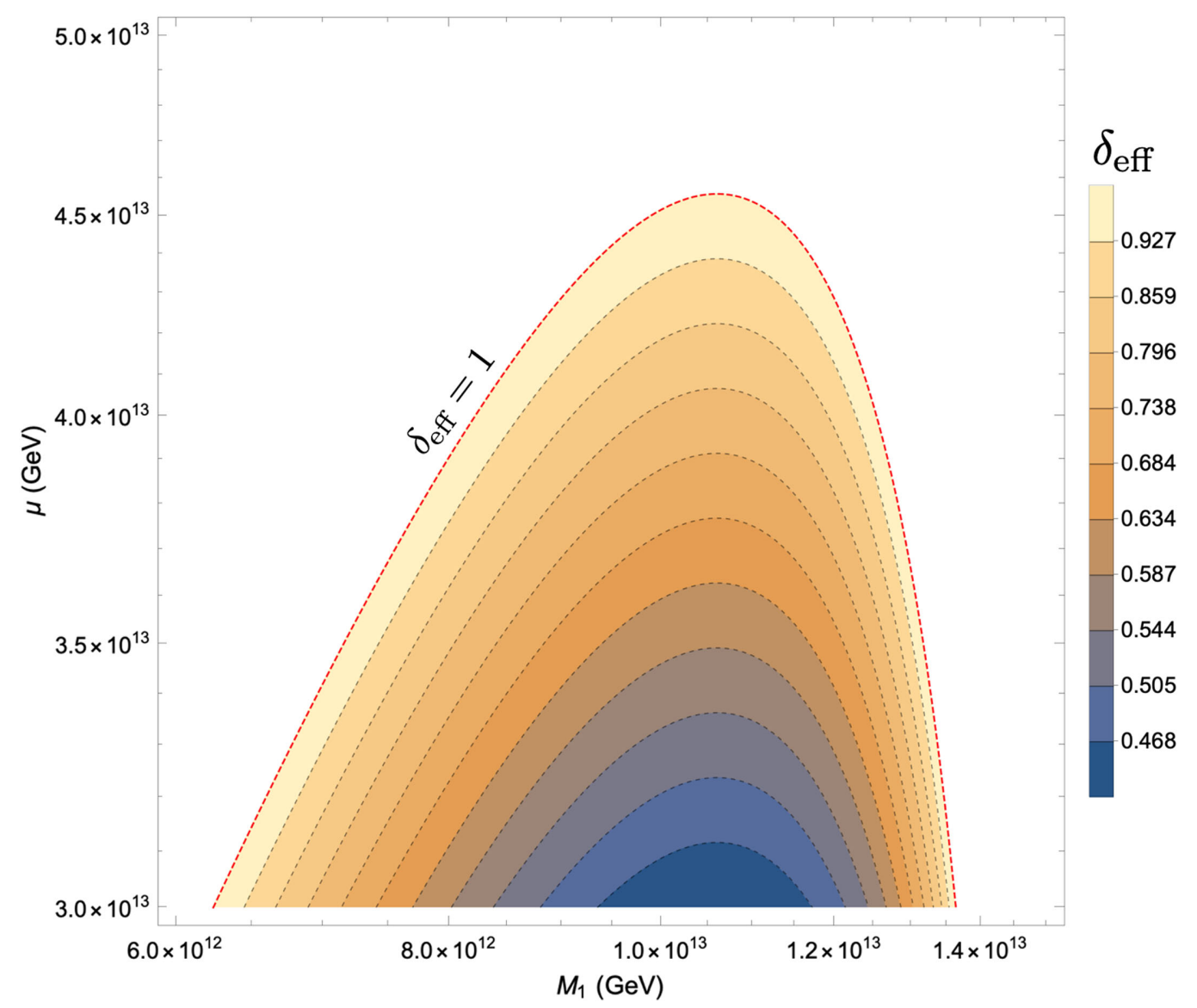

FIG. 6. Range of right-handed neutrino masses $M_{1}$ and the $\mu$ term satisfying the baryon asymmetry $Y_{B} \simeq 8.7 \times 10^{-11}$, with $m_{\nu_{2}} \simeq$ $0.0086 \mathrm{eV}$ in the models $\mathrm{C}-1$ or C-2. The red-dashed line corresponds to limit of maximal $C P$-violating phase $\delta_{\text {eff }}$.

In order to ensure that the lightest right-handed neutrino decays immediately out-of-equilibrium, we need to satisfy the constraint $\Gamma_{L_{\alpha} h}>\Gamma_{2 N_{1}}$. The decay rate of the lightest right-handed neutrino $N_{1}$ is given by

$$
\Gamma_{L_{\alpha} h}=\frac{\left(y y^{\dagger}\right)_{11}}{4 \pi} M_{1} \equiv \frac{\left|y_{1}\right|^{2}}{4 \pi} M_{1}=\frac{m_{\nu_{1}} M_{1}^{2}}{4 \pi v^{2} \sin ^{2} \beta},
$$

where we have included decays to $L_{\alpha} h$ and $\bar{L}_{\alpha} \bar{h}$ and we denoted the dominant contribution of $\left(y y^{\dagger}\right)_{11} \simeq\left|y_{1}\right|^{2}$. If we now compare the decay rate (71) to the inflaton decay rate into the right-handed neutrinos (65), we find

$$
m_{\nu_{1}} \gtrsim 1.6 \times 10^{-12} \mathrm{eV}
$$

which is clearly easily satisfied.

\section{WESS-ZUMINO-TYPE MODELS OF INFLATION}

In this section, we consider models based on the WessZumino superpotential (4); supersymmetry breaking is most easily attained by simply adding a constant, $\tilde{m}$, to the superpotential, giving $m_{3 / 2}=\tilde{m}$. Indeed if the constant is promoted to a Polonyi term in $W$, then the inflationary potential is affected, and it becomes difficult to maintain a flat potential at large field values $[21,26,27]$ with high-scale supersymmetry breaking.

In the absence of a Polonyi term, using only a constant term in $W$, supersymmetry breaking is generated by an $F$ term for $T$. Gaugino masses are given by Eq. (10) upon replacing $z \rightarrow T$. However, in this case, because the VEV of $T$ is of the order of the Planck scale $M_{P}$, even if we write $f=f_{0}+f_{1} T / \Lambda_{T}$, if $\Lambda_{T} \ll\langle T\rangle$, we are inevitably led to $m_{1 / 2} \sim m_{3 / 2}$ since we must require $f_{1}\langle T\rangle / \Lambda_{T} \lesssim\left(1 / g^{2}\right)$. Thus, there is no simple way to realize a high-scale supersymmetry model with $m_{1 / 2} \sim m_{0}>m>m_{3 / 2}$. Therefore, we no longer consider WZ-1 models with $m_{3 / 2}<m$.

For WZ-2 models with $m_{3 / 2}>m$, the above problems are no longer present, as we can again break supersymmetry with a constant superpotential term, with $m_{1 / 2} \sim$ $m_{0} \sim m_{3 / 2}>m$. The constant term $\tilde{m}$, which breaks supersymmetry, does not shift the minimum, and at the end of inflation, we are left with $\left\langle T_{R}\right\rangle=1 / 2,\left\langle T_{I}\right\rangle=0$, and $\left\langle\phi_{R}\right\rangle=\left\langle\phi_{I}\right\rangle=0$. As in the case of the $\mathrm{C}-2$ models, we can no longer consider the gravitino as a dark matter candidate. 


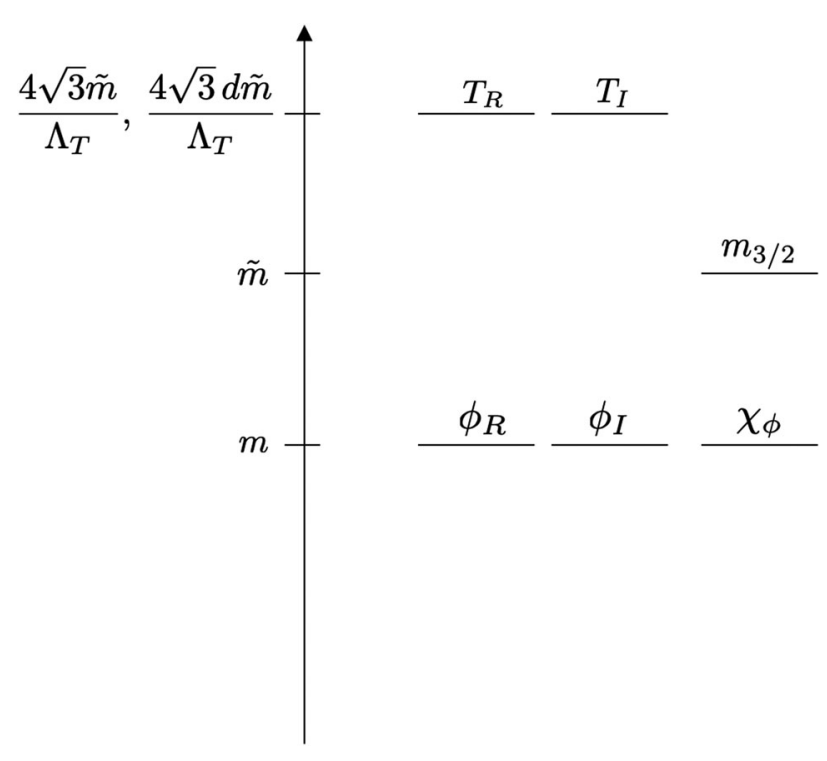

FIG. 7. Mass spectrum of the model WZ-2. In this scenario, $\phi_{R}$ is identified as the inflaton, and $m_{\phi_{R}} \simeq m<m_{3 / 2}$.

The mass spectrum for the Wess-Zumino type models is relatively simple. Both the real and imaginary parts of the inflaton have a common mass,

$$
m_{\phi_{R, I}}^{2} \simeq m^{2} .
$$

In order to ensure the stability of the potential during inflation, the $T$ field is dynamically stabilized with the higher-order terms in Eq. (7). As in the case of the strongly stabilized Polonyi field, the stabilization of the volume modulus $T$ results in a mass, which is hierarchically higher than the gravitino mass,

$$
m_{T_{R}}^{2} \simeq \frac{48 m_{3 / 2}^{2}}{\Lambda_{T}^{2}}, \quad m_{T_{I}}^{2} \simeq \frac{48 d^{2} m_{3 / 2}^{2}}{\Lambda_{T}^{2}},
$$

where the constant $d$ was define in Eq. (9). As we start with only two superfields in this case, and supersymmetry is broken, there is only one chiral fermion which is associated with the inflaton. Namely, the inflatino mass is simply

$$
m_{\chi_{\phi}} \simeq m
$$

The fermion associated with $T$ is the Goldstino and becomes the longitudinal component of the gravitino and $m_{3 / 2}=\tilde{m}$. The mass spectrum for WZ-2 is illustrated in Fig. 7.

\section{LEPTOGENESIS IN WESS-ZUMINO-TYPE MODELS}

A key difference between the Cecotti and Wess-Zumino models of inflation in no-scale supergravity is the manner in which the Universe reheats [26]. In models where the inflaton is associated the volume modulus, $T$, the inflaton couples to Standard Model fields (and their supersymmetric partners). Thus, there are many open decay channels leading to reheating. In high-scale supersymmetry, as discussed earlier, the dominant decay mode is the twobody decay to two Higgs bosons. However, in WessZumino models, where the inflaton is associated with a matterlike field $\phi$, in the absence of a direct coupling of the inflaton to Standard Model fields, there are no decay channels available $[26,95]$. Reheating in this case typically relies on a coupling of the inflaton in the gauge kinetic function which then allows for decays to gauge bosons (and in the case of low scale supersymmetry, to gauginos) $[26,78,95]$.

In Wess-Zumino-like models, it is, however, possible to associate the inflaton with a right-handed sneutrino [16]. ${ }^{6}$ For example, starting with the Wess-Zumino superpotential (4), we can equate $\phi$ with $N_{2}$ (or $N_{3}$, however, we still require $2 M_{1} \lesssim m$ for leptogenesis, so $\phi$ can not be $N_{1}$ ). Thus, we consider the superpotential,

$$
W \supset-m\left(\frac{N_{2}^{3}}{3 \sqrt{3}}\right)+y_{i \alpha} N_{i} L_{\alpha} H_{u}+\frac{1}{2} N_{i} M_{i} N_{i},
$$

where $i=1,2,3$. From Eq. (76), we see that there is a direct coupling of the inflaton to $L_{\alpha}$ and $H_{u}$ with Yukawa coupling $y_{2 \alpha}$. To preserve the form of the Starobinsky potential, we must require $M_{2}=m$. For leptogenesis, we require a decay of the inflaton to $N_{1}$ and assume the mass hierarchy $2 M_{1} \lesssim m<M_{3}$.

In models of weak scale supersymmetry, the Yukawa coupling of the inflaton to $L_{\alpha} H_{u}$ leads to efficient reheating after inflation [16]. However, in high-scale supersymmetry with $\tilde{m}>m$, the two possible tree level decays (slepton + Higgs, or lepton + Higgsino) are both kinematically forbidden. One loop decays to Standard Model fields are possible, but these are suppressed. It is, however, possible to introduce a superpotential coupling,

$$
W \supset-\frac{1}{2} \kappa N_{2} N_{1} N_{1}
$$

which leads to the following trilinear term in the Lagrangian:

$$
\mathcal{L} \supset-\kappa \tilde{N}_{2} N_{1} N_{1}+\text { H.c. },
$$

\footnotetext{
${ }^{6}$ The association of the matterlike field $\phi$ with $N$ is not possible in Cecotti-like models. When supersymmetry is broken, $\phi$ gets a VEV given by Eq. (33b), which approaches the Planck scale in high-scale supersymmetric models. This VEV induces a bilinear R-parity violating term, which induces gravitino decay and is strongly constrained in order for the gravitino lifetime to remain sufficiently long [55,92]. This bound translates into $y_{\nu} \lesssim 10^{-21}$, thus preventing it in generating a neutrino mass and lepton asymmetry.
} 


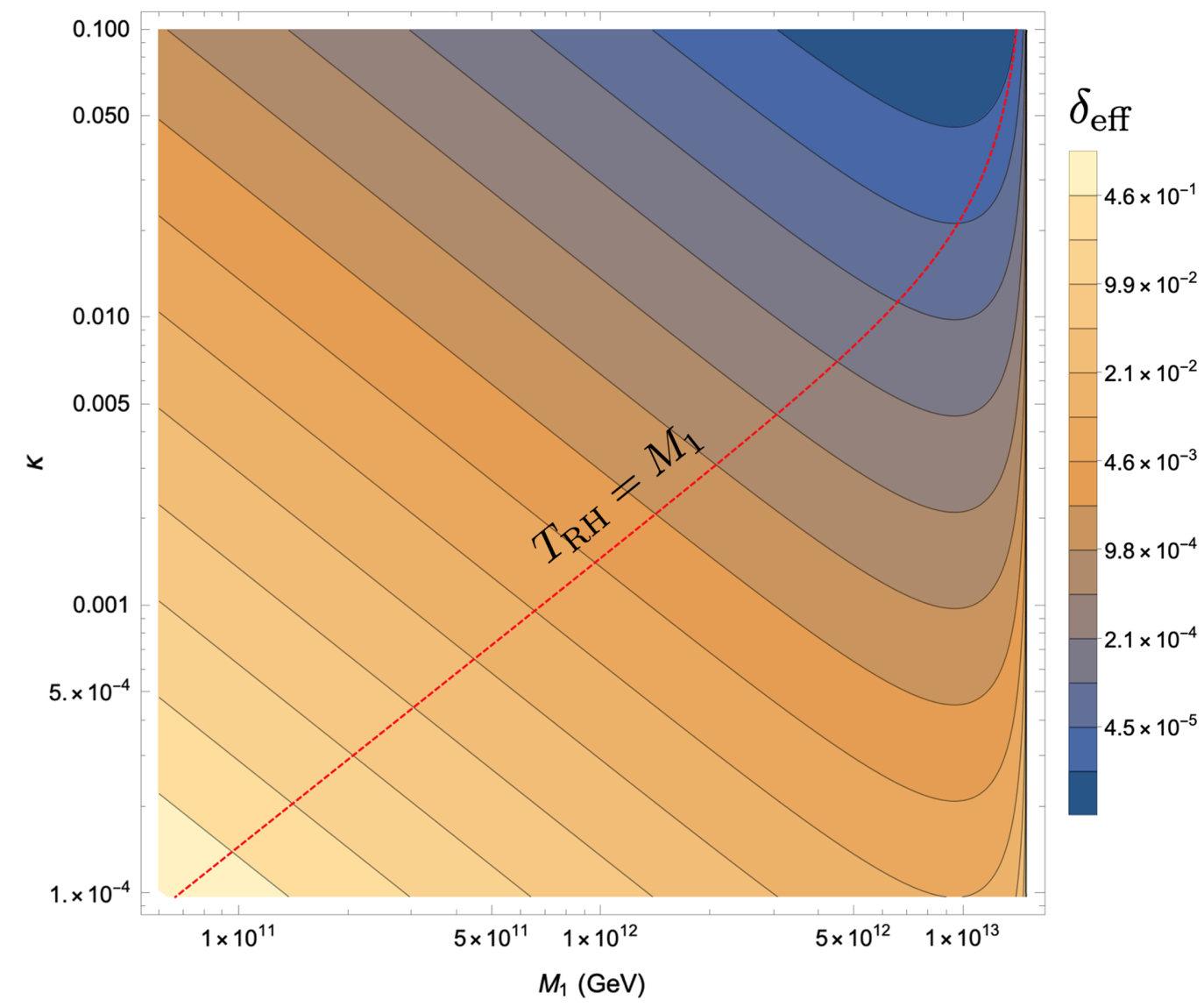

FIG. 8. $C P$-violating effective phase $\delta_{\text {eff }}$ as a function of the lightest right-handed neutrino mass $M_{1}$ and the trilinear coupling $\kappa$ for $m_{\nu_{3}} \simeq 0.05 \mathrm{eV}$ for the model WZ-2. The area below the red-dashed line shows the region when $T_{\mathrm{RH}} \lesssim M_{1}$, which is necessary for nonthermal leptogenesis.

which leads to the following decay rate for the inflaton to two lighter right-handed neutrinos:

$$
\Gamma_{2 N_{1}}=\frac{\kappa^{2} m}{8 \pi}\left(1-\frac{4 M_{1}^{2}}{m^{2}}\right)^{3 / 2} .
$$

This decay to two right-handed neutrinos, $N_{1}$, dominates and is responsible for generating reheating and leptogenesis. The reheat temperature is given by Eq. (61) with the substitution $\Gamma_{2 h} \rightarrow \Gamma_{2 N_{1}}$. We can express the reheating temperature as

$T_{R H} \simeq 6.8 \times 10^{14} \kappa \mathrm{GeV}\left(\frac{m}{3 \times 10^{13} \mathrm{GeV}}\right)^{1 / 2}\left(1-\frac{4 M_{1}^{2}}{m^{2}}\right)^{3 / 4}$.

Assuming noninstantaneous reheating, the number density of $n_{N 1}$ is again given by Eq. (67) with $N=2$. We find that the number density to entropy ratio is

$\frac{n_{N_{1}}}{s}=\frac{5 T_{\mathrm{RH}}}{2 m} \simeq 57 \kappa\left(\frac{m}{3 \times 10^{13} \mathrm{GeV}}\right)^{-1 / 2}\left(1-\frac{4 M_{1}^{2}}{m^{2}}\right)^{3 / 4}$, and we can express the baryon asymmetry (31) as

$$
\begin{aligned}
Y_{B} \simeq & 4 \times 10^{-3} \delta_{\mathrm{eff}} \kappa\left(\frac{m_{\nu_{i}}}{0.05 \mathrm{eV}}\right)\left(\frac{M_{1}}{10^{12} \mathrm{GeV}}\right) \\
& \times\left(\frac{m}{3 \times 10^{13} \mathrm{GeV}}\right)^{-1 / 2}\left(1-\frac{4 M_{1}^{2}}{m^{2}}\right)^{3 / 4} .
\end{aligned}
$$

If we connect it to the observed baryon asymmetry of the Universe, $Y_{B} \simeq 8.7 \times 10^{-11}$, we obtain the following constraint:

$$
\begin{aligned}
& \delta_{\mathrm{eff}} \kappa\left(\frac{m_{\nu_{i}}}{0.05 \mathrm{eV}}\right)\left(\frac{M_{1}}{10^{12} \mathrm{GeV}}\right)\left(\frac{m}{3 \times 10^{13} \mathrm{GeV}}\right)^{-1 / 2} \\
& \times\left(1-\frac{4 M_{1}^{2}}{m^{2}}\right)^{3 / 4} \simeq 2.2 \times 10^{-8} .
\end{aligned}
$$

For fixed $m_{\nu}$ and $m$, the constraint in Eq. (83) on the $\left(\kappa, M_{1}, \delta_{\text {eff }}\right)$ parameter space is plotted in Figs. 8 and 9 . For each pair $\left(M_{1}, \kappa\right)$, the shading determines the required value of $\delta_{\text {eff }}$ needed to obtain the correct baryon asymmetry. Also plotted is the boundary for which $T_{\mathrm{RH}}=M_{1}$. For values of $\kappa$ above this line, $T_{\mathrm{RH}}>M_{1}$, and one must consider 


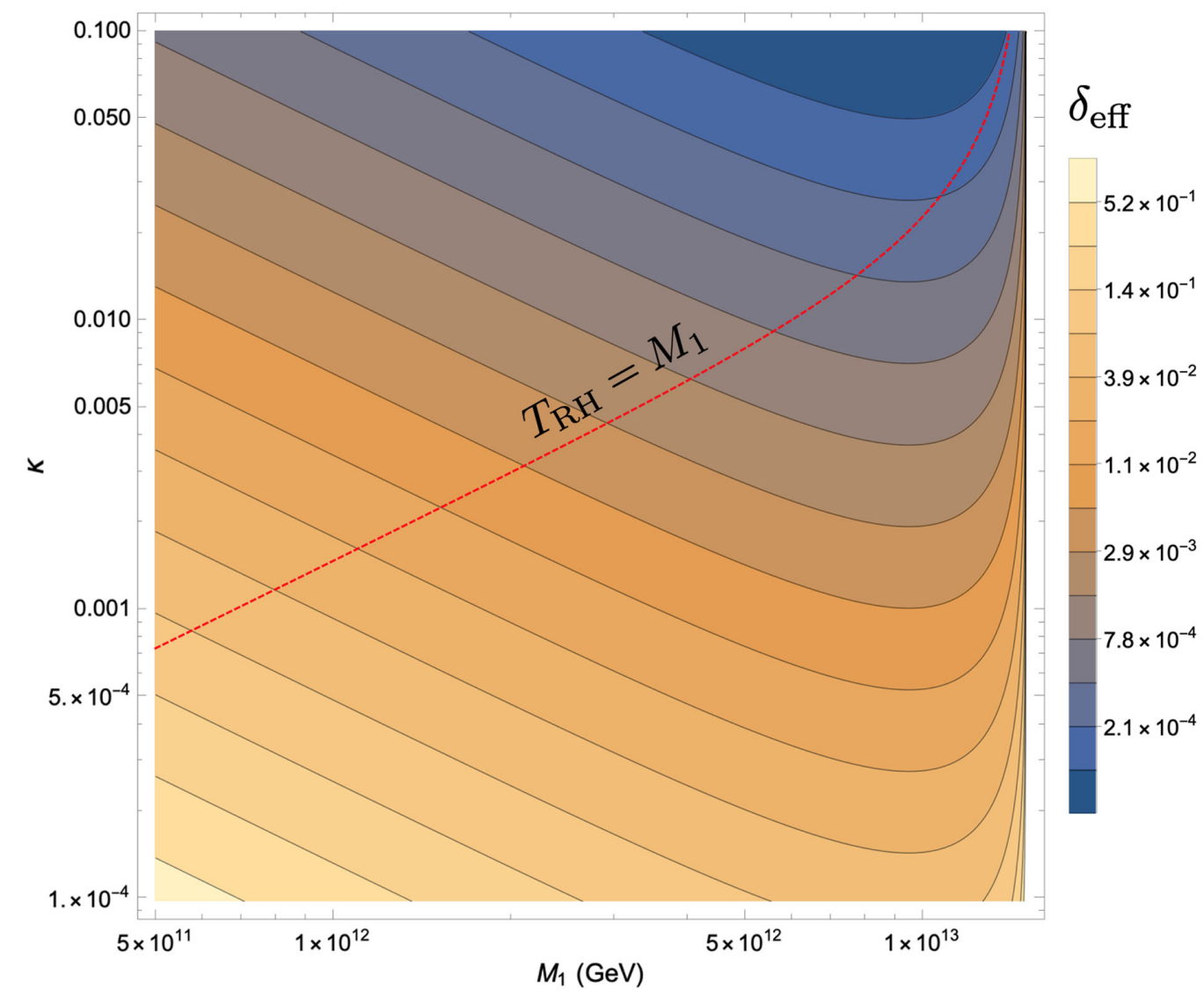

FIG. 9. $C P$-violating effective phase $\delta_{\text {eff }}$ as a function of the lightest right-handed neutrino mass $M_{1}$ and the Yukawa coupling $\kappa$ for $m_{\nu_{2}} \simeq 0.0086 \mathrm{eV}$ for the model WZ-2. The area below the red-dashed line shows the region when $T_{\mathrm{RH}} \lesssim M_{1}$, which is necessary for nonthermal leptogenesis.

thermal leptogenesis [96]. As one can see from the figures, this is a relatively efficient mechanism for generating the baryon asymmetry, though it does not require particularly small couplings or phases.

Finally, we compare the decay rate of the lightest righthanded neutrino $N_{1}$ with the inflaton decay rate as a check on its out-of-equilibrium decay. The decay rate for $N_{1}$ is again given by Eq. (71) and must be compared with the inflaton decay rate in Eq. (79). Requiring $\Gamma_{L_{\alpha} h} \gtrsim \Gamma_{2 N_{1}}$, we find

$$
\frac{m_{\nu_{1}} M_{1}^{2}}{\left\langle H_{u}\right\rangle^{2}} \gtrsim \frac{1}{2} \kappa^{2} m\left(1-\frac{4 M_{1}^{2}}{m^{2}}\right)^{3 / 2}
$$

or

$$
m_{\nu_{1}} \gtrsim 230 \kappa^{2} \mathrm{eV}\left(\frac{M_{1}}{10^{12} \mathrm{GeV}}\right)^{-2}\left(1-\frac{4 M_{1}^{2}}{m^{2}}\right)^{3 / 2} .
$$

The strongest bound on $m_{\nu_{1}}$ is found when $\kappa$ is set at its maximum value, which occurs when $T_{\mathrm{RH}}=M_{1}$ or when $M_{1}=6.8 \times 10^{14} \kappa \mathrm{GeV}$. In this case, $m_{\nu_{1}} \gtrsim 5 \times 10^{-4} \mathrm{eV}$. For fixed $M_{1}$, values of $\kappa$ lower than the value needed for
$T_{\mathrm{RH}}=M_{1}$ reduce this lower bound on $m_{\nu_{1}}$. This limit is always satisfied for the inverted neutrino hierarchy.

\section{CONCLUSIONS}

Viable models of the very early Universe must account for both inflation and the generation of net baryon asymmetry. In the models presented here, although both of these mechanisms were operative near the scale of grand unification, neither are explicitly dependent on a specific model of grand unification. Furthermore, while these models are inherently supersymmetric, supersymmetry is broken at a scale above the inflationary scale of $m=$ $3 \times 10^{13} \mathrm{GeV}$ and aside from the possible role of the gravitino (if $m_{3 / 2}<m$ ), supersymmetry does not affect the low energy behavior of the theory.

The models of inflation we consider are based on noscale supergravity. The inflationary sector requires two chiral superfields [15], which parametrizes a noncompact $\frac{S U(2,1)}{S U(2) \times U(1)}$ coset manifold. In a particular basis, one of the fields may be associated with the volume modulus while the second appears as a matterlike field. Due to the underlying symmetry of the theory, either of the fields 
can play the role of the inflaton $[14,15,18]$ whose potential can take the form of the Starobinsky potential [11]. However, once supersymmetry is broken, and couplings to the Standard Model are introduced, these two classes of inflationary models appear quite different.

Supersymmetry breaking can be achieved by simply adding a constant term to the superpotential $[26,75]$, by introducing a Polonyi sector [74], or in the case of a matterlike inflaton, by adding a linear term to the superpotential [21]. We have derived the conditions for which the inflationary potential allows for inflation in the presence of high-scale supersymmetry breaking. For modulus-driven inflation, adding a constant to the superpotential perturbs the potential in such a way so as to always lead to an AdS vacuum [26]. Adding a Polonyi sector, preserves the form of the potential suitable for inflation. This is true independent of the scale of supersymmetry breaking $\tilde{m}$, although as we have shown, for $\tilde{m}>m$, additional superpotential couplings are needed. In the case of a matterlike inflaton, a linear term or a Polonyi sector severely perturbs the inflationary potential for high-scale supersymmetry breaking. In contrast, in this case, adding a constant term allows an arbitrarily high supersymmetry breaking scale. We have also derived the mass spectrum in the inflationary/susy breaking sector when the supersymmetry breaking scale approaches and exceeds the inflaton mass. We have shown that there is no inflatino problem in these models.

Reheating in the two classes of inflationary models is also quite different. The volume modulus couples to all sectors of the theory, and in the case of high-scale supersymmetry breaking, final state Higgs bosons are the dominant decay mode $[26,27,55,56]$. In contrast, without an explicit superpotential coupling to the Standard Model, reheating for a matterlike inflaton occurs only if inflaton couples to the gauge sector through the gauge kinetic function $[26,78,95]$. As a consequence, we have here associated the inflaton with one of the right-handed sneutrinos.

Subsequent to reheating, we have considered, in detail, mechanisms for leptogenesis [67]. Assuming the existence of a right-handed neutrino sector, we have assumed that one of the Majorana right-handed neutrinos are lighter than the inflationary scale, $M_{1} \lesssim m$. In the case of modulus-driven inflation, we have derived the branching ratios for the production of right-handed neutrinos from inflaton decay during reheating. Once produced during reheating, the right-handed neutrinos decay quickly (their decay rate is faster than their production rate so long as the lightest left-handed neutrino has $m_{\nu_{1}}>3.3 \times 10^{-12} \mathrm{eV}$ ) and decay out-of-equilibrium if $T_{\mathrm{RH}}<M_{1}$. The resulting lepton asymmetry is then converted to a baryon asymmetry via sphaleron interactions.

In the case of a matterlike inflaton, we have found that we must associate one of heavier two right-handed sneutrinos with the inflaton. A coupling of the inflaton leads to decays predominantly into the lightest right-handed neutrino, and its out-of-equilibrium decay simultaneously reheats the Universe and produces the lepton asymmetry. In this case, the departure from thermal equilibrium requires $m_{\nu_{1}}>10^{-3} \mathrm{eV}$.

In both cases of a moduluslike and matterlike inflaton, we distinguish between a supersymmetry breaking scale in which the gravitino mass is above or below the inflationary scale. In both cases, we have derived the mass spectra of the inflationary/SUSY breaking sectors. When $m_{3 / 2}<m$, the gravitino may be the dark matter [54]. A hierarchy between the gravitino mass and the supersymmetry breaking scale is possible with the aid of a strongly stabilized Polonyi sector [76]. Thus it is only possible for modulusdriven inflation. When $m_{3 / 2}>m$, both types of inflationary models are viable, but a new dark matter candidate is needed. Integrating such a candidate will be the subject of future work.

\section{ACKNOWLEDGMENTS}

This work was supported by the France-US PICS MicroDark and by Institut Pascal at Universit ParisSaclay with the support of the P2I and SPU research departments and the P2IO Laboratory of Excellence (program Investissements d'avenir ANR-11-IDEX-0003-01 Paris-Saclay and ANR-10-LABX-0038), as well as the IPhT. Y.M. acknowledges partial support from the European Union Horizon 2020 research and innovation programme under the Marie Sklodowska-Curie: RISE InvisiblesPlus (Grant Agreement No. 690575), the ITN Elusives (Grant Agreement No. 674896) and the Red Consolider MultiDark . The work of K. K., K. A. O., and S. V. was supported in part by the DOE Grant No. DESC0011842 at the University of Minnesota. K. A. O. acknowledges support by the Director, Office of Science, Office of High Energy Physics of the U.S. Department of Energy under the Contract No. DE-AC02-05CH11231. K. A. O. would also like to thank the Department of Physics and the high energy theory group at the University of California, Berkeley as well as the theory group at LBNL for their hospitality and financial support while finishing this work. 
[1] L. Maiani, AIP Conf. Proc. C7909031, 1 (1979); Recent Developments in Gauge Theories, Proceedings of the Nato Advanced Study Institute, Cargese, France, 1979, edited by G. 't Hooft et al., Nato Advanced Study Institutes Series Series B, Physics Vol. 59 (Plenum press, New York, USA, 1980); E. Witten, Phys. Lett. B 105, 267 (1981).

[2] J. R. Ellis, S. Kelley, and D. V. Nanopoulos, Phys. Lett. B 249, 441 (1990); 260, 131 (1991); U. Amaldi, W. de Boer, and H. Furstenau, Phys. Lett. B 260, 447 (1991); P. Langacker and M.-X. Luo, Phys. Rev. D 44, 817 (1991); C. Giunti, C. W. Kim, and U. W. Lee, Mod. Phys. Lett. A 06, 1745 (1991).

[3] J. R. Ellis and D. Ross, Phys. Lett. B 506, 331 (2001).

[4] L. E. Ibanez and G. G. Ross, Phys. Lett. B 110, 215 (1982); K. Inoue, A. Kakuto, H. Komatsu, and S. Takeshita, Prog. Theor. Phys. 68, 927 (1982); 70, 330(E) (1983); Prog. Theor. Phys. 70, 330 (1983); L. E. Ibanez, Phys. Lett. B 118, 73 (1982); J. R. Ellis, D. V. Nanopoulos, and K. Tamvakis, Phys. Lett. B 121, 123 (1983); J. R. Ellis, J. S. Hagelin, D. V. Nanopoulos, and K. Tamvakis, Phys. Lett. B 125, 275 (1983); L. Alvarez-Gaume, J. Polchinski, and M. B. Wise, Nucl. Phys. B221, 495 (1983).

[5] H. Goldberg, Phys. Rev. Lett. 50, 1419 (1983); J. Ellis, J. Hagelin, D. Nanopoulos, K. Olive, and M. Srednicki, Nucl. Phys. B238, 453 (1984).

[6] J. R. Ellis, D. V. Nanopoulos, K. A. Olive, and K. Tamvakis, Phys. Lett. B 118, 335 (1982); Nucl. Phys. B221, 52 (1983); K. Nakayama and F. Takahashi, J. Cosmol. Astropart. Phys. 10 (2011) 033.

[7] D. V. Nanopoulos, K. A. Olive, M. Srednicki, and K. Tamvakis, Phys. Lett. B 123, 41 (1983); R. Holman, P. Ramond, and G. G. Ross, Phys. Lett. B 137, 343 (1984); A. B. Goncharov and A. D. Linde, Phys. Lett. B 139, 27 (1984).

[8] E. J. Copeland, A. R. Liddle, D. H. Lyth, E. D. Stewart, and D. Wands, Phys. Rev. D 49, 6410 (1994); E. D. Stewart, Phys. Rev. D 51, 6847 (1995).

[9] E. Cremmer, S. Ferrara, C. Kounnas, and D. V. Nanopoulos, Phys. Lett. B 133, 61 (1983); J. R. Ellis, A. B. Lahanas, D. V. Nanopoulos, and K. Tamvakis, Phys. Lett. B 134, 429 (1984); A. B. Lahanas and D. V. Nanopoulos, Phys. Rep. 145, 1 (1987).

[10] A.S. Goncharov and A. D. Linde, Classical Quantum Gravity 1, L75 (1984); C. Kounnas and M. Quiros, Phys. Lett. B 151, 189 (1985); J. R. Ellis, K. Enqvist, D. V. Nanopoulos, K. A. Olive, and M. Srednicki, Phys. Lett. B 152, 175 (1985); 156, 452(E) (1985).

[11] A. A. Starobinsky, Phys. Lett. B 91, 99 (1980).

[12] V. F. Mukhanov and G. V. Chibisov, Pis'ma Zh. Eksp. Teor. Fiz. 33, 549 (1981) [JETP Lett. 33, 532 (1981)], http://www .jetpletters.ac.ru/ps/1510/article_23079.shtml.

[13] N. Aghanim et al. (Planck Collaboration), arXiv: 1807.06209; Y. Akrami et al. (Planck Collaboration), arXiv:1807.06211.

[14] J. Ellis, D. V. Nanopoulos, and K. A. Olive, Phys. Rev. Lett. 111, 111301 (2013).

[15] J. Ellis, D. V. Nanopoulos, and K. A. Olive, J. Cosmol. Astropart. Phys. 10 (2013) 009.

[16] J. Ellis, D. V. Nanopoulos, and K. A. Olive, Phys. Rev. D 89, 043502 (2014).
[17] J. Ellis, D. V. Nanopoulos, and K. A. Olive, Phys. Rev. D 97 , 043530 (2018).

[18] J. Ellis, D. V. Nanopoulos, K. A. Olive, and S. Verner, J. High Energy Phys. 03 (2019) 099.

[19] J. Ellis, D. V. Nanopoulos, K. A. Olive, and S. Verner, Phys. Rev. D 100, 025009 (2019).

[20] J. Ellis, D. V. Nanopoulos, K. A. Olive, and S. Verner, J. Cosmol. Astropart. Phys. 09 (2019) 040.

[21] M. C. Romao and S. F. King, J. High Energy Phys. 07 (2017) 033; S. F. King and E. Perdomo, J. High Energy Phys. 05 (2019) 211.

[22] R. Kallosh and A. Linde, J. Cosmol. Astropart. Phys. 06 (2013) 028.

[23] F. Farakos, A. Kehagias, and A. Riotto, Nucl. Phys. B876, 187 (2013).

[24] S. Ferrara, A. Kehagias, and A. Riotto, Fortschr. Phys. 62, 573 (2014); 63, 2 (2015); R. Kallosh, A. Linde, B. Vercnocke, and W. Chemissany, J. Cosmol. Astropart. Phys. 07 (2014) 053; K. Hamaguchi, T. Moroi, and T. Terada, Phys. Lett. B 733, 305 (2014); J. Ellis, M. A. G. García, D. V. Nanopoulos, and K. A. Olive, J. Cosmol. Astropart. Phys. 05 (2014) 037; 08 (2014) 044.

[25] I. Antoniadis, E. Dudas, S. Ferrara, and A. Sagnotti, Phys. Lett. B 733, 32 (2014).

[26] J. Ellis, M. A. G. Garcia, D. V. Nanopoulos, and K. A. Olive, J. Cosmol. Astropart. Phys. 10 (2015) 003.

[27] E. Dudas, T. Gherghetta, Y. Mambrini, and K. A. Olive, Phys. Rev. D 96, 115032 (2017).

[28] S. Ferrara, R. Kallosh, A. Linde, and M. Porrati, Phys. Rev. D 88, 085038 (2013); W. Buchmüller, V. Domcke, and C. Wieck, Phys. Lett. B 730, 155 (2014); C. Pallis, J. Cosmol. Astropart. Phys. 04 (2014) 024; 08 (2014) 057; W. Buchmuller, E. Dudas, L. Heurtier, and C. Wieck, J. High Energy Phys. 09 (2014) 053; J. Ellis, M. A. G. García, D. V. Nanopoulos, and K. A. Olive, J. Cosmol. Astropart. Phys. 01 (2015) 010; T. Terada, Y. Watanabe, Y. Yamada, and J. Yokoyama, J. High Energy Phys. 02 (2015) 105; W. Buchmuller, E. Dudas, L. Heurtier, A. Westphal, C. Wieck, and M. W. Winkler, J. High Energy Phys. 04 (2015) 058; A. B. Lahanas and K. Tamvakis, Phys. Rev. D 91, 085001 (2015); I. Dalianis and F. Farakos, J. Cosmol. Astropart. Phys. 07 (2015) 044; I. Garg and S. Mohanty, Phys. Lett. B 751, 7 (2015); J. Ellis, M. A. G. García, D. V. Nanopoulos, and K. A. Olive, J. Cosmol. Astropart. Phys. 07 (2015) 050; E. Dudas and C. Wieck, J. High Energy Phys. 10 (2015) 062; M. Scalisi, J. High Energy Phys. 12 (2015) 134; S. Ferrara, A. Kehagias, and M. Porrati, J. High Energy Phys. 08 (2015) 001; J. Ellis, M. A. G. García, D. V. Nanopoulos, and K. A. Olive, Classical Quantum Gravity 33, 094001 (2016); A. Addazi and M. Y. Khlopov, Phys. Lett. B 766, 17 (2017); C. Pallis and N. Toumbas, Adv. High Energy Phys. 2017, 6759267 (2017); T. Kobayashi, O. Seto, and T. H. Tatsuishi, Prog. Theor. Exp. Phys. 2017, 123B04 (2017); I. Garg and S. Mohanty, Int. J. Mod. Phys. A 33, 1850127 (2018); W. Ahmed and A. Karozas, Phys. Rev. D 98, 023538 (2018); Y. Cai, R. Deen, B. A. Ovrut, and A. Purves, J. High Energy Phys. 09 (2018) 001; S. Khalil, A. Moursy, A. K. Saha, and A. Sil, Phys. Rev. D 99, 095022 (2019).

[29] J. Ellis, M. A. G. Garcia, N. Nagata, D. V. Nanopoulos, and K. A. Olive, J. Cosmol. Astropart. Phys. 11 (2016) 018. 
[30] J. Ellis, M. A. G. Garcia, N. Nagata, D. V. Nanopoulos, and K. A. Olive, J. Cosmol. Astropart. Phys. 07 (2017) 006; 04 (2019) 009.

[31] S. A. R. Ellis, T. Gherghetta, K. Kaneta, and K. A. Olive, Phys. Rev. D 98, 055009 (2018).

[32] H. Georgi and D. V. Nanopoulos, Nucl. Phys. B159, 16 (1979); C. E. Vayonakis, Phys. Lett. B 82, 224 (1979); A. Masiero, Phys. Lett. B 93, 295 (1980); Q. Shafi, M. Sondermann, and C. Wetterich, Phys. Lett. B 92, 304 (1980); F. del Aguila and L. E. Ibanez, Nucl. Phys. B177, 60 (1981); R. N. Mohapatra and G. Senjanovic, Phys. Rev. D 27, 1601 (1983); M. Fukugita and T. Yanagida, Physics of neutrinos, in Physics and Astrophysics of Neutrinos, edited by M. Fukugita and A. Suzuki (Springer, New York, 1994), pp. 1-248 (Kyoto University, Report No. YITP-K-1050, 1994); and Kyoto Univ. Report No. YITP-K-1050 (93/12,rec.Feb.94) 248 p. C.

[33] Y. Mambrini, K. A. Olive, J. Quevillon, and B. Zaldivar, Phys. Rev. Lett. 110, 241306 (2013).

[34] Y. Mambrini, N. Nagata, K. A. Olive, J. Quevillon, and J. Zheng, Phys. Rev. D 91, 095010 (2015).

[35] N. Nagata, K. A. Olive, and J. Zheng, J. High Energy Phys. 10 (2015) 193.

[36] Y. Mambrini, N. Nagata, K. A. Olive, and J. Zheng, Phys. Rev. D 93, 111703 (2016).

[37] H. Pagels and J. R. Primack, Phys. Rev. Lett. 48, 223 (1982).

[38] D. V. Nanopoulos, K. A. Olive, and M. Srednicki, Phys. Lett. B 127, 30 (1983).

[39] K. A. Olive, D. N. Schramm, and M. Srednicki, Nucl. Phys. B255, 495 (1985).

[40] J. R. Ellis, K. A. Olive, Y. Santoso, and V. C. Spanos, Phys. Lett. B 588, 7 (2004).

[41] J. L. Feng, S. F. Su, and F. Takayama, Phys. Rev. D 70, 063514 (2004); J. L. Feng, S. Su, and F. Takayama, Phys. Rev. D 70, 075019 (2004).

[42] F. D. Steffen, J. Cosmol. Astropart. Phys. 09 (2006) 001.

[43] W. Buchmuller, L. Covi, K. Hamaguchi, A. Ibarra, and T. Yanagida, J. High Energy Phys. 03 (2007) 037; W. Buchmuller, AIP Conf. Proc. 1200, 155 (2010).

[44] S. Bailly, K. Y. Choi, K. Jedamzik, and L. Roszkowski, J. High Energy Phys. 05 (2009) 103.

[45] L. Covi, J. Hasenkamp, S. Pokorski, and J. Roberts, J. High Energy Phys. 11 (2009) 003.

[46] M. Aaboud et al. (ATLAS Collaboration), J. High Energy Phys. 06 (2018) 107; Phys. Rev. D 97, 112001 (2018); A. M. Sirunyan et al. (CMS Collaboration), Eur. Phys. J. C 77, 710 (2017); J. High Energy Phys. 05 (2018) 025.

[47] D. S. Akerib et al. (LUX Collaboration), Phys. Rev. Lett. 118, 021303 (2017).

[48] X. Cui et al. (PandaX-II Collaboration), Phys. Rev. Lett. 119, 181302 (2017).

[49] E. Aprile et al. (XENON Collaboration), Phys. Rev. Lett. 121, 111302 (2018).

[50] J. Ellis, J. L. Evans, F. Luo, N. Nagata, K. A. Olive, and P. Sandick, Eur. Phys. J. C 76, 8 (2016); J. Ellis, J. L. Evans, A. Mustafayev, N. Nagata, and K. A. Olive, Eur. Phys. J. C 76, 592 (2016); J. Ellis, J. L. Evans, N. Nagata, D. V. Nanopoulos, and K. A. Olive, Eur. Phys. J. C 77, 232 (2017); J. Ellis, J. L. Evans, F. Luo, K. A. Olive, and
J. Zheng, Eur. Phys. J. C 78, 425 (2018); E. Bagnaschi et al., Eur. Phys. J. C 79, 149 (2019).

[51] M. Ibe, T. Moroi, and T. T. Yanagida, Phys. Lett. B 644, 355 (2007); M. Ibe and T. T. Yanagida, Phys. Lett. B 709, 374 (2012); M. Ibe, S. Matsumoto, and T. T. Yanagida, Phys. Rev. D 85, 095011 (2012); J. L. Evans and K. A. Olive, Phys. Rev. D 90, 115020 (2014); J. L. Evans, M. Ibe, K. A. Olive, and T. T. Yanagida, Phys. Rev. D 91, 055008 (2015); J. L. Evans, N. Nagata, and K. A. Olive, Eur. Phys. J. C 79, 490 (2019).

[52] J. L. Evans, M. Ibe, K. A. Olive, and T. T. Yanagida, Eur. Phys. J. C 73, 2468 (2013); J. L. Evans, K. A. Olive, M. Ibe, and T. T. Yanagida, Eur. Phys. J. C 73, 2611 (2013).

[53] G. F. Giudice and A. Strumia, Nucl. Phys. B858, 63 (2012); E. Bagnaschi, G. F. Giudice, P. Slavich, and A. Strumia, J. High Energy Phys. 09 (2014) 092.

[54] E. Dudas, Y. Mambrini, and K. Olive, Phys. Rev. Lett. 119, 051801 (2017).

[55] E. Dudas, T. Gherghetta, K. Kaneta, Y. Mambrini, and K. A. Olive, Phys. Rev. D 98, 015030 (2018).

[56] K. Kaneta, Y. Mambrini, and K. A. Olive, Phys. Rev. D 99, 063508 (2019).

[57] J. R. Ellis, J. E. Kim, and D. V. Nanopoulos, Phys. Lett. B 145, 181 (1984).

[58] T. Moroi, arXiv:hep-ph/9503210.

[59] J. R. Ellis, D. V. Nanopoulos, K. A. Olive, and S. J. Rey, Astropart. Phys. 4, 371 (1996).

[60] G. F. Giudice, A. Riotto, and I. Tkachev, J. High Energy Phys. 11 (1999) 036.

[61] M. Bolz, A. Brandenburg, and W. Buchmuller, Nucl. Phys. B606, 518 (2001); B790, 336(E) (2008); J. Pradler and F. D. Steffen, Phys. Rev. D 75, 023509 (2007); V. S. Rychkov and A. Strumia, Phys. Rev. D 75, 075011 (2007).

[62] J. Ellis, M. A. G. Garcia, D. V. Nanopoulos, K. A. Olive, and M. Peloso, J. Cosmol. Astropart. Phys. 03 (2016) 008.

[63] K. Benakli, Y. Chen, E. Dudas, and Y. Mambrini, Phys. Rev. D 95, 095002 (2017).

[64] M. A. G. Garcia, Y. Mambrini, K. A. Olive, and M. Peloso, Phys. Rev. D 96, 103510 (2017).

[65] M. Kadastik, K. Kannike, and M. Raidal, Phys. Rev. D 80, 085020 (2009); 81, 029903(E) (2010); 81, 015002 (2010); M. Frigerio and T. Hambye, Phys. Rev. D 81, 075002 (2010).

[66] J. L. Evans, N. Nagata, K. A. Olive, and J. Zheng, J. High Energy Phys. 02 (2016) 120.

[67] M. Fukugita and T. Yanagida, Phys. Lett. B 174, 45 (1986).

[68] S. Davidson and A. Ibarra, Phys. Lett. B 535, 25 (2002); E. Nardi, Y. Nir, E. Roulet, and J. Racker, J. High Energy Phys. 01 (2006) 164; A. Abada, S. Davidson, A. Ibarra, F.-X. Josse-Michaux, M. Losada, and A. Riotto, J. High Energy Phys. 09 (2006) 010; R. Barbieri, P. Creminelli, A. Strumia, and N. Tetradis, Nucl. Phys. B575, 61 (2000); M. Raidal, A. Strumia, and K. Turzynski, Phys. Lett. B 609, 351 (2005); 632, 752(E) (2006); A. Pilaftsis and T. E. J. Underwood, Nucl. Phys. B692, 303 (2004); P. S. B. Dev, P. Millington, A. Pilaftsis, and D. Teresi, Nucl. Phys. B886, 569 (2014); W. Buchmuller, V. Domcke, and K. Schmitz, Nucl. Phys. B862, 587 (2012).

[69] P. Minkowski, Phys. Lett. B 67, 421 (1977); M. Gell-Mann, P. Ramond, and R. Slansky, in Supergravity, edited by 
D. Freedman and P. Van Nieuwenhuizen (North Holland, Amsterdam, 1979), pp. 315-321. ISBN 044485438x; T. Yanagida, AIP Conf. Proc. C7902131, 95 (1979); R. N. Mohapatra and G. Senjanovic, Phys. Rev. Lett. 44, 912 (1980); J. Schechter and J. W. F. Valle, Phys. Rev. D 22, 2227 (1980); 25, 774 (1982).

[70] V. A. Kuzmin, V. A. Rubakov, and M. E. Shaposhnikov, Phys. Lett. B 155, 36 (1985).

[71] S. Y. Khlebnikov and M. E. Shaposhnikov, Nucl. Phys. B308, 885 (1988); J. A. Harvey and M. S. Turner, Phys. Rev. D 42, 3344 (1990).

[72] S. Cecotti, Phys. Lett. B 190, 86 (1987).

[73] J. R. Ellis, C. Kounnas, and D. V. Nanopoulos, Phys. Lett. B 143, 410 (1984).

[74] J. Polonyi, Budapest Report No. KFKI-1977-93, 1977.

[75] J. Ellis, B. Nagaraj, D. V. Nanopoulos, and K. A. Olive, J. High Energy Phys. 11 (2018) 110; J. Ellis, B. Nagaraj, D. V. Nanopoulos, K. A. Olive, and S. Verner, J. High Energy Phys. 10 (2019) 161.

[76] M. Dine, R. Kitano, A. Morisse, and Y. Shirman, Phys. Rev. D 73, 123518 (2006); R. Kitano, Phys. Lett. B 641, 203 (2006); R. Kallosh and A. D. Linde, J. High Energy Phys. 02 (2007) 002; H. Abe, T. Higaki, and T. Kobayashi, Phys. Rev. D 76, 105003 (2007); J. Fan, M. Reece, and L.-T. Wang, J. High Energy Phys. 09 (2011) 126.

[77] E. Dudas, C. Papineau, and S. Pokorski, J. High Energy Phys. 02 (2007) 028; H. Abe, T. Higaki, T. Kobayashi, and Y. Omura, Phys. Rev. D 75, 025019 (2007).

[78] R. Kallosh, A. Linde, K. A. Olive, and T. Rube, Phys. Rev. D 84, 083519 (2011); A. Linde, Y. Mambrini, and K. A. Olive, Phys. Rev. D 85, 066005 (2012).

[79] E. Dudas, A. Linde, Y. Mambrini, A. Mustafayev, and K. A. Olive, Eur. Phys. J. C 73, 2268 (2013).

[80] K. Nakayama, F. Takahashi, and T. T. Yanagida, Phys. Lett. B 718, 526 (2012).
[81] M. A. G. Garcia and K. A. Olive, J. Cosmol. Astropart. Phys. 09 (2013) 007.

[82] J. L. Evans, M. A. G. Garcia, and K. A. Olive, J. Cosmol. Astropart. Phys. 03 (2014) 022.

[83] P. H. Frampton, S. L. Glashow, and T. Yanagida, Phys. Lett. B 548, 119 (2002).

[84] M. Tanabashi et al. (Particle Data Group), Phys. Rev. D 98, 030001 (2018).

[85] M. A. Luty, Phys. Rev. D 45, 455 (1992).

[86] L. Covi, E. Roulet, and F. Vissani, Phys. Lett. B 384, 169 (1996); M. Flanz, E. A. Paschos, and U. Sarkar, Phys. Lett. B 345, 248 (1995); 384, 487(E) (1996); 382, 447(E) (1996).

[87] B. A. Campbell, S. Davidson, and K. A. Olive, Phys. Lett. B 303, 63 (1993); Nucl. Phys. B399, 111 (1993).

[88] G. F. Giudice, M. Peloso, A. Riotto, and I. Tkachev, J. High Energy Phys. 08 (1999) 014.

[89] T. Asaka, K. Hamaguchi, M. Kawasaki, and T. Yanagida, Phys. Lett. B 464, 12 (1999).

[90] T. Asaka, K. Hamaguchi, M. Kawasaki, and T. Yanagida, Phys. Rev. D 61, 083512 (2000).

[91] J. Ellis, M. A. G. Garcia, N. Nagata, D. V. Nanopoulos, and K. A. Olive, Phys. Lett. B 797, 134864 (2019); arXiv: 1910.11755.

[92] E. Dudas, T. Gherghetta, K. Kaneta, Y. Mambrini, and K. A. Olive, Phys. Rev. D 100, 035004 (2019).

[93] S. Ferrara and A. Van Proeyen, Fortschr. Phys. 64, 896 (2016).

[94] H. P. Nilles, K. A. Olive, and M. Peloso, Phys. Lett. B 522 , 304 (2001).

[95] M. Endo, K. Kadota, K. A. Olive, F. Takahashi, and T. T. Yanagida, J. Cosmol. Astropart. Phys. 02 (2007) 018.

[96] G. F. Giudice, A. Notari, M. Raidal, A. Riotto, and A. Strumia, Nucl. Phys. B685, 89 (2004). 